\title{
Exact results on the large-scale stochastic transport of inertial particles including the Basset history term
}

Boi, S.

2019-06

Boi , S 2019 , ' Exact results on the large-scale stochastic transport of inertial particles including the Basset history term ' , Physics of Fluids , vol. 31 , no. 6 , 063304 . https://doi.org/10.1063/1.5092987

http://hdl.handle.net/10138/305545

https://doi.org/10.1063/1.5092987

unspecified

acceptedVersion

Downloaded from Helda, University of Helsinki institutional repository.

This is an electronic reprint of the original article.

This reprint may differ from the original in pagination and typographic detail.

Please cite the original version. 


\section{Exact results on the large-scale stochastic transport of inertial particles including the Basset history term}

S. Boi ${ }^{1, a)}$

${ }^{1}$ Department of Mathematics and Statistics, University of Helsinki, Gustaf Hällströmin katu 2b, Helsinki, Finland

(Dated: 10 May 2019)

Maxey-Riley equation and its simplified versions represent the most widespread tool to investigate dynamics and dispersion of inertial small particles in turbulent flows. Numerical solution of such models is often very challenging, and some of their terms, such as the molecular diffusivity or the Basset history force, are often neglected to reduce the complexity upon suitable approximations. Here we propose exact results with regard to the rate of transport on large time scales in random shear flows. These can be expediently used as a benchmark to develop and assess algorithms when solving this class of stochastic integro-differential problems on large time scales.

a) Electronic mail: simone.boi@helsinki.fi 


\section{INTRODUCTION}

The study of the dynamics of small particles in a fluid flow where their inertia cannot be neglected has been a topic of paramount importance in many research fields. These range from theoretical problems arising in statistical mechanics ${ }^{1}$ to applications such as the radiation scattering in atmosphere ${ }^{2}$, astrophysics ${ }^{3,4}$, droplet dynamics in rain initiation ${ }^{5-7}$, plasma physics ${ }^{8-10}$, and biological oceanography ${ }^{11}$. To tackle these problems, the starting points are the old Basset-Boussinesq-Oseen equation ${ }^{1,12,13}$ and the modern, more rigorous, and well-known Maxey-Riley equation ${ }^{14-16}$ (in short, BBO and MR respectively). The former is still often used when suitable hypotheses are fulfilled, thanks to its simplicity in comparison to the latter. Moreover, the infamous Basset history force term is sometimes neglected in turn, as its slow time decay makes it computationally expensive to handle ${ }^{17-21}$. We refer to Refs. 13 and 22 as to the validity, the usages, and the approximations of the several models.

Besides establishing a model for the inertial particle dynamics, the other important ingredient in this category of problems corresponds to the type of carrier flows and the regime one wants to analyze. In this sense, the stochastic construction of turbulent synthetic models is a typical tool to produce particle-laden flows when investigating small-scale turbulence and turbulent dispersion. This technique indeed renders it possible to control the statistical properties of turbulence better than by other methods based on Direct Numerical Simulations (DNS) or chaotic flows. The advantage of having more controllability justifies why synthetic models are oftentimes utilized although some key differences from DNS are present, especially when it comes to particle transport ${ }^{23-26}$.

Within this context, in the past decade the study of dispersion in the presence of white noise and stochastic flows mimicking turbulence also took place in literature, in a growing demand of more and more sophisticated, general, and rigorous numerical models ${ }^{23,27-29}$. On the one hand, the role of the molecular Brownian motion can indeed be crucial for the particle dynamics in a flow; it can enhance or hinder the transport via interference mechanisms ${ }^{30}$. In most extreme scenarios, the presence of a small molecular diffusivity can trigger standard diffusion from a trapped configuration (e.g. in cellular flows) or, conversely, regularize superdiffusive regimes ${ }^{31,32}$. On the other hand, the Basset history force can be as determinant in creating ballistic diffusion regimes in flows where dispersion would be normal otherwise ${ }^{33,34}$. It is therefore natural that in plenty of situations one is interested 
in accounting for the effects of all these terms and their mutual interplay, which cannot be deemed trivial in general.

However, there is no closed-form solution for the Maxey-Riley equation, due to the complexity induced by the very Basset history force, which features slow decay and nonlocality with respect to time ${ }^{27}$. Although well-established numerical schemes are available for the pure deterministic Maxey-Riley equation ${ }^{27,35}$, its solution in the presence of white noise entails Monte Carlo techniques to generate the trajectories. The strong patchiness of such trajectories can make the convergence difficult to achieve when the Basset term is present. This occurs in that the methods for ordinary differential equations have a lower accuracy order when stochastic processes enter into play. For example, it is well known that the common, explicit Euler method is of order $O(\sqrt{\Delta t})$ in the presence of white noise rather than $O(\Delta t)$. Higher order algorithms, for instance 4-th order Runge-Kutta methods, are in general different and more complex than a trivial application of the ones for deterministic $\mathrm{ODEs}^{36}$. Moreover, the Basset term puts things into an even more complicated situation. As already said previously, that term in fact is a slowly decaying convolution integral with respect to the past trajectory history, and it is usually approximated by means of exponential interpolating functions ${ }^{35}$. When one has to cope with both Brownian trajectories and stochastic flows together, it is therefore useful to have a benchmark whatsoever. One is indeed given the opportunity to compare it to results from possible numerical schemes adapted to the stochastic case, or even new approaches.

The aim of the present article therefore is to provide an exactly evaluable quantity for the most common models of inertial particles - to wit, Basset-Boussinesq-Oseen and MaxeyRiley. Namely, we will compute the so-called eddy diffusivity tensor ${ }^{30,37-39}$, the large-time transport rate for asymptotic, standard diffusion (i.e., the mean square displacement of the particle evolving linearly as a function of time) for a well-known class of exactly solvable flows in these problems, that is the class of the shear flows ${ }^{40}$. For such a category, we will reduce calculating the eddy-diffusivity tensor to the evaluation of double integrals. Unlike the original stochastic integro-differential problem, the latter can be numerically carried out by standard and robust techniques implemented in widespread commercial softwares too. Our flow will be a stochastic one having well-defined statistical properties set a priori, in a similar manner to synthetic models for turbulence ${ }^{23,24,26}$. The value of the eddy diffusivity will thus be dependent on the very statistical properties of the shear flow, namely its time- 
space correlation function.

With such an approach, we thus achieve a twofold goal. The first is to give an insight about the interaction between Basset history force, Brownian motion, and other possible refinement terms in the Maxey-Riley equation, albeit for a toy model such as the random parallel flow. As we will see, those terms engender contributions to the transport which are not trivial at all even in a simple case like that. The second is to provide an exact test case for algorithms solving $\mathrm{BBO}$ or $\mathrm{MR}$ when white noise is included, specifically in asymptotic-time regimes. This in practice entails to compute the eddy diffusivity and exploit it as a benchmark to assess the accuracy of algorithms or schemes employed in numerical simulations on long time scales. Such a test would be just a first step in the validation of the scheme, along with other possible comparisons verifying the performance at short time or in more complex flows than the random shear geometry, for which analytical solution are not available. In this framework, given any resolution scheme, on the one hand it is always possible to generate a random shear flow whose statistical properties are the ones considered in this work. On the other hand, it is always possible to contrast the eddy diffusivity deriving from Monte Carlo trajectories with the analytical result contained in this work. This approach is actionable regardless of the values of the parameters involved in the $\mathrm{BBO}$ or $\mathrm{MR}$ equations.

In Sec. II we will algebrically manipulate the BBO model in order to calculate the eddy diffusivity for a random shear flow, and will depict and table the resulting numbers for some significant parameter values both of the model and the velocity Eulerian correlation function. The steps involved to obtain the results for this case will be preparatory for Sec. III, where these calculations will be reused and extended so as to obtain the eddy diffusivity of the full MR equation. Again, results will be depicted for some sample but significant parameter choices. This should give us a full insight about the involved dynamics. Sec. IV will be dedicated to the conclusions, and details about the computation steps and how to generate random shear flows with the correlation functions considered herein will be postponed to the Appendix. 


\section{GENERAL PROPERTIES OF THE BASSET-BOUSSINESQ-OSEEN EQUATION}

The Maxey-Riley model in the original Basset-Boussinesq-Oseen approximation ${ }^{12,14}$, supplemented by an additive white noise ${ }^{38}$, can be written in the following dimensionless form:

$$
\begin{aligned}
\frac{d \boldsymbol{\xi}}{d t} & =\boldsymbol{v}(t) \\
\frac{d \boldsymbol{v}}{d t} & =-\frac{\boldsymbol{v}-\boldsymbol{u}(\boldsymbol{\xi}(t), t)}{\mathrm{St}}+\beta \frac{d \boldsymbol{u}(\boldsymbol{\xi}(t), t)}{d t}+\frac{\sqrt{2 D_{0}}}{\mathrm{St}} \boldsymbol{\eta}(t) \\
& +\sqrt{\frac{3 \beta}{\pi \mathrm{St}}} \int_{0}^{t} d t^{\prime} \sqrt{\frac{1}{t-t^{\prime}}} \frac{d}{d t^{\prime}}\left[\boldsymbol{u}\left(t^{\prime}\right)-\boldsymbol{v}\left(\boldsymbol{\xi}\left(t^{\prime}\right), t^{\prime}\right)\right]
\end{aligned}
$$

where $\boldsymbol{\xi}$ is the particle coordinate, $\boldsymbol{v}$ is the particle velocity, $\beta=\frac{3 \rho_{f}}{\rho_{f}+2 \rho_{p}}, 0 \leqslant \beta \leqslant 3$, with $\rho_{f}$ and $\rho_{p}$ being the fluid and particle (mass) density, $\frac{\mathrm{d}}{\mathrm{d} t}=\partial_{t}+\boldsymbol{v} \cdot \boldsymbol{\nabla}, \boldsymbol{\eta}$ is a Gaussian white noise (i.e. the time derivative of a $d$-dimensional Wiener process), $D_{0}$ is the dimensionless molecular diffusivity (i.e. the Péclet number) and, finally, $\mathrm{St}=\frac{r_{p}^{2}}{3 \nu \beta T}$ is the Stokes number. In the latter definition, $T$ is a typical time of the carrier flow $\boldsymbol{u}, \nu$ is the fluid kinematic viscosity and $r_{p}$ is the particle radius. The first addend on the left hand side in the particle acceleration equation constitutes the Stokes viscous drag. The second addend is the added mass effect caused by the pressure gradient. The third is the molecular Brownian motion and the fourth is the well-known Basset history force, which depends on the past trajectory of the particle in the flow. Eq. (1) is valid under a number of physical hypotheses which we recall here briefly ${ }^{12,14}$. It assumes low particle Reynolds number (that is, $\max \mid \boldsymbol{u}(\xi(t), t)-$ $\left.\boldsymbol{v}(t) \mid r_{p} / \nu \ll 1\right)$, small Stokes numbers and small particle radius in comparison to the typical flow wavelength (i.e., $r_{p} / L \ll 1$ ). All these hypotheses implicate that the particle is passively advected in the flow, which means there is no feedback effect of the particle on the flow in turn. The particle experiences a Stokes flow at any time, thus making many nonlinear terms negligible in the Navier-Stokes equation. In addition to that, the approximation $\operatorname{St} \beta \ll 1$ is made to have no significant difference between material derivatives along the trajectory of the particle and following the fluid ${ }^{14}$. We finally need to impose the further condition $\boldsymbol{v}(0)=\boldsymbol{u}(\boldsymbol{\xi}(0), 0)$ for the validity of this form of the Basset integral term ${ }^{41}$. Generalizations of the latter are possible, but those will not be relevant for us. This is because they just introduce transients depending on the initial conditions, whereas we are going to analyze 
the asymptotic transport ${ }^{22}$. We can thus safely stick to the original Basset history term expression.

To start our analysis, it is convenient to take the Laplace transform (which here will be denoted by $\hat{\bullet}$ ) of both sides of Eq. (1) and recast the several terms in the following form:

$$
\hat{\boldsymbol{v}}(s)=\hat{\boldsymbol{\sigma}}(s)+\hat{K}_{0}(s) \hat{\boldsymbol{u}}(s)+\sqrt{2 D_{0}} \hat{K}_{1}(s) \hat{\boldsymbol{\eta}}(s)
$$

where have introduced the functions:

$$
\hat{\boldsymbol{\sigma}}(s)=\frac{(1-\beta) \boldsymbol{u}(\boldsymbol{\xi}(0))}{s+\frac{1}{\mathrm{St}}+\sqrt{\frac{3 \beta s}{\mathrm{St}}}} \quad \hat{K}_{0}(s)=\frac{\beta s+\frac{1}{\mathrm{St}}+\sqrt{\frac{3 \beta s}{\mathrm{St}}}}{s+\frac{1}{\mathrm{St}}+\sqrt{\frac{3 \beta s}{\mathrm{St}}}} \quad \hat{K}_{1}(s)=\frac{\frac{1}{\mathrm{St}}}{s+\frac{1}{\mathrm{St}}+\sqrt{\frac{3 \beta s}{\mathrm{St}}}}
$$

bearing in mind the initial condition $\boldsymbol{v}(0)=\boldsymbol{u}(\boldsymbol{\xi}(0), 0)$. From the above expressions the following relationship easily follows:

$$
\hat{K}_{0}(s)=1+(\beta-1) \operatorname{St} \hat{K}_{1}(s) s
$$

Also note that:

$$
\hat{K}_{0}(0)=\hat{K}_{1}(0)=1
$$

The role and the importance of the latter observation will become clearer in Sec. II A. By computing the inverse Laplace transform of (2), we get the following compact form of Eq. (1):

$$
\boldsymbol{v}(t)=\boldsymbol{\sigma}\left(\boldsymbol{\xi}_{0}, \boldsymbol{v}_{0}, t\right)+\int_{0}^{t} K_{0}\left(t-t^{\prime}\right) \boldsymbol{u}\left(\boldsymbol{\xi}\left(t^{\prime}\right), t^{\prime}\right) d t^{\prime}+\sqrt{2 D_{0}} \int_{0}^{t} K_{1}\left(t-t^{\prime}\right) \boldsymbol{\eta}\left(t^{\prime}\right) d t^{\prime}
$$

It is now evident that the functions $K_{0}, K_{1}$ are nothing but Green functions characterizing the linear response of the dynamical system (1), similarly to those commonly encountered in statistical mechanics. The function $\boldsymbol{\sigma}$ instead is a transient term depending on the initial condition, which does not play any role into the asymptotic dynamics. By direct computation (see Appendixes A-C for details) one can arrive at the explicit expressions for $K_{0}(t)$ and $K_{1}(t)$ :

$$
K_{1}(t)=\frac{1}{\operatorname{St}\left(A_{2}-A_{1}\right)}\left(A_{2} e^{A_{2}^{2} t} \operatorname{erfc}\left(A_{2} \sqrt{t}\right)-A_{1} e^{A_{1}^{2} t} \operatorname{erfc}\left(A_{1} \sqrt{t}\right)\right)
$$

where erfc is the complementary Euler error function and $A_{1}$ and $A_{2}$ are given in Appendixes A and C. The expression for $K_{0}(t)$ follows from Eq. (4):

$$
\begin{aligned}
K_{0}(t) & =\delta(t)+(\beta-1)+\operatorname{St} K_{1}^{\prime}(t) \\
& =\delta(t)+\frac{\beta-1}{A_{2}-A_{1}}\left(A_{2}^{3} e^{A_{2}^{2} t} \operatorname{erfc}\left(A_{2} \sqrt{t}\right)-A_{1}^{3} e^{A_{1}^{2} t} \operatorname{erfc}\left(A_{1} \sqrt{t}\right)\right)
\end{aligned}
$$


Noteworthy, the properties we have determined up to this point are completely general; we have not specified the type of external flow yet. The particular case of heavy particles (i.e., $\beta=0$ ), where the Basset term disappears and $A_{1}, A_{2}$ are imaginary and complex conjugates, has already been analyzed in Ref. 40.

\section{A. Eddy diffusivity for shear flows: effect of the Basset history term}

Our aim at this point is to exploit and generalize achievements of Ref. 40 in order to obtain first-principle results (i.e. following from the ruling equation (1) without approximations) with regard to the large-scale transport of inertial particles. The large-scale transport regime we are going to consider here is the one characterized by the so-called eddy diffusivity ${ }^{37,42}$ :

$$
\mathbb{D}=\lim _{t \rightarrow \infty} \frac{1}{2 t}\left\langle\delta \boldsymbol{\xi}\left(t+t_{0}\right) \otimes \delta \boldsymbol{\xi}\left(t_{0}\right)+\delta \boldsymbol{\xi}\left(t_{0}\right) \otimes \delta \boldsymbol{\xi}\left(t+t_{0}\right)\right\rangle
$$

where $\delta \boldsymbol{\xi}(t)=\boldsymbol{\xi}(t)-\langle\boldsymbol{\xi}(t)\rangle$ and the average is meant over the sample space of the Brownian motion and the one of the stochastic flow or the initial conditions of the particles ${ }^{31}$. The definition above goes under the assumption that the diffusion is standard (that is, the variance of the mean square displacement grows asymptotically like $\propto t$ ), and that quantity itself is independent of the initial conditions. The latter statement is equivalent to say that Lagrangian correlation functions (and the diffusion consequently) are statistically stationary, at least over long time scales.

For tracer particles (i.e., St $=0$ ), the eddy diffusivity can be obtained by means of Taylor's 1922 celebrated formula ${ }^{43}$ :

$$
\mathbb{D}=D_{0} \mathbb{I}+\lim _{t \rightarrow \infty} \frac{1}{2} \int_{0}^{t} d s\langle\boldsymbol{u}(\boldsymbol{\xi}(t), t) \otimes \boldsymbol{u}(\boldsymbol{\xi}(s), s)+\boldsymbol{u}(\boldsymbol{\xi}(s), s) \otimes \boldsymbol{u}(\boldsymbol{\xi}(t), t)\rangle
$$

Remarkably, when we have (asymptotic) stationarity, the correlation function decays sufficiently fast and Eq. (5) is fulfilled, the same Taylor formula formally holds for many more general models of particle dynamics, including the one in Eq. (1) - see also Ref. 22 for a rigorous illustration. Our large-scale transport will thus be completely defined in terms of the eddy-diffusivity tensor $\mathbb{D}$, the expression of which reads formally identical too Eq. (10). Of course the key point is that the trajectories over which the Lagrangian correlation 
functions are evaluated are dramatically different in the tracer case and the inertial particles we are considering here. They are indeed results of different dynamical models. Obtaining an analytical expression for $\mathbb{D}$ by exploiting the ruling equation (1) is the main aim of this section.

For general carriers flows this problem can be only tackled via numerical techniques. This is a consequence of the character of Eq. (1), which is nonlinear and nonlocal with respect to time. These difficulties can be reduced and handled for the class of shear flows:

$$
\boldsymbol{u}(\boldsymbol{x}, t)=u_{1}\left(x_{2}, \ldots, x_{d}\right) \boldsymbol{e}_{1}
$$

where $\boldsymbol{e}_{1}=(1,0, \ldots, 0)$ is the constant unit vector associated to the first axis. This simple geometry readily enforces the incompressibility condition.

According to the hypotheses of Taylor's formula, we need to evaluate the correlation function of the flow field over the particle trajectories $\left\langle\boldsymbol{u}\left(\boldsymbol{\xi}\left(t_{1}\right), t_{1}\right) \otimes \boldsymbol{u}\left(\boldsymbol{\xi}\left(t_{2}\right), t_{2}\right)\right\rangle$ and verify that the process is asymptotically stationary. That is, for a finite constant $t=t_{2}-t_{1}$, we should get:

$$
\lim _{t_{1} \rightarrow \infty}\left\langle\boldsymbol{u}\left(\boldsymbol{\xi}\left(t_{1}\right), t_{1}\right) \otimes \boldsymbol{u}\left(\boldsymbol{\xi}\left(t_{1}+t\right), t_{1}+t\right)\right\rangle=\mathbb{C}(|t|)
$$

If this happens, then the eddy diffusivity is given by:

$$
\mathrm{D}=D_{0} \mathbb{I}+\int_{0}^{\infty} \mathbb{C}(t) d t
$$

The remarkable point is that for the shear flow (11) such an expression can be calculated on analytical ground. Indeed, along the directions $n \neq 1$ the flow components are zero and Eq. (6) reduces to:

$$
v_{i}(t)=\sqrt{2 D_{0}} \int_{0}^{t} K_{1}\left(t-t^{\prime}\right) \eta_{i}\left(t^{\prime}\right) d t^{\prime},
$$

In these directions, the transient term $\boldsymbol{\sigma}\left(\boldsymbol{\xi}_{0}, \boldsymbol{v}_{0}, t\right)$ in Eq. (6) is identically zero due to the absence of flow.

We now need to define the nature of the shear flow. Our choice is for a zero-mean Gaussian random field having a homogeneous and stationary Eulerian correlation function. In plain symbols:

$$
\begin{aligned}
\langle\boldsymbol{u}(\boldsymbol{x}, t)\rangle & =0 \\
\left\langle\boldsymbol{u}\left(\boldsymbol{x}_{1}, t_{1}\right) \otimes \boldsymbol{u}\left(\boldsymbol{x}_{2}, t_{2}\right)\right\rangle & =B\left(\boldsymbol{x}_{1}-\boldsymbol{x}_{2},\left|t_{1}-t_{2}\right|\right) \boldsymbol{e}_{1} \otimes \boldsymbol{e}_{1}
\end{aligned}
$$


From the above expressions, we can get the Lagrangian correlations in Fourier space (Fourier transformed fields are denoted by $\check{c}^{\prime}$ ):

$$
\begin{aligned}
& \left\langle\boldsymbol{u}\left(\boldsymbol{\xi}\left(t_{1}\right), t_{1}\right) \otimes \boldsymbol{u}\left(\boldsymbol{\xi}\left(t_{2}\right), t_{2}\right)\right\rangle \\
& =\int \frac{d \boldsymbol{k}_{1}^{d-1}}{(2 \pi)^{d-1}} \int \frac{d \boldsymbol{k}_{2}^{d-1}}{(2 \pi)^{d-1}}\left\langle e^{i\left(\boldsymbol{k}_{1} \cdot \boldsymbol{\xi}\left(t_{1}\right)+\boldsymbol{k}_{2} \cdot \boldsymbol{\xi}\left(t_{2}\right)\right)} \check{\boldsymbol{u}}\left(\boldsymbol{k}_{1}, t_{1}\right) \otimes \check{\boldsymbol{u}}\left(\boldsymbol{k}_{2}, t_{2}\right)\right\rangle \\
& =\int \frac{d \boldsymbol{k}_{1}^{d-1}}{(2 \pi)^{d-1}} \int \frac{d \boldsymbol{k}_{2}^{d-1}}{(2 \pi)^{d-1}}\left\langle e^{i\left(\boldsymbol{k}_{1} \cdot \boldsymbol{\xi}\left(t_{1}\right)+\boldsymbol{k}_{2} \cdot \boldsymbol{\xi}\left(t_{2}\right)\right)}\right\rangle\left\langle\check{\boldsymbol{u}}\left(\boldsymbol{k}_{1}, t_{1}\right) \otimes \check{\boldsymbol{u}}\left(\boldsymbol{k}_{2}, t_{2}\right)\right\rangle \\
& =\int \frac{d \boldsymbol{k}^{d-1}}{(2 \pi)^{d-1}}\left\langle e^{i \boldsymbol{k} \cdot\left(\boldsymbol{\xi}\left(t_{1}\right)-\boldsymbol{\xi}\left(t_{2}\right)\right)}\right\rangle \check{B}\left(\boldsymbol{k},\left|t_{2}-t_{1}\right|\right) \boldsymbol{e}_{1} \otimes \boldsymbol{e}_{1}
\end{aligned}
$$

In the above steps we made use of the independence between the flow and the trajectories, with the former pointing solely in the direction $n=1$ and the latter being affected only by the molecular diffusion. We also exploited the homogeneity of the flow, i.e.:

$$
\left\langle\check{\boldsymbol{u}}\left(\boldsymbol{k}_{1}, t_{1}\right) \otimes \check{\boldsymbol{u}}\left(\boldsymbol{k}_{2}, t_{2}\right)\right\rangle=\check{B}\left(\boldsymbol{k}_{1},\left|t_{2}-t_{1}\right|\right) \delta\left(\boldsymbol{k}_{2}-\boldsymbol{k}_{1}\right) \boldsymbol{e}_{1} \otimes \boldsymbol{e}_{1}
$$

In the imaginary exponential above, we see from Eq. (11) that only the directions $n \neq 1$ play a role, and from Eq. (13):

$$
\xi_{i}(t)=\sqrt{2 D_{0}} \int_{0}^{t} G(t-s) \eta_{i} d s, \quad G(t-s)=\int_{s}^{t} K_{1}\left(s^{\prime}-s\right) d s^{\prime}
$$

The last ingredient we need is to compute the average in Eq. (15) exploiting the first expression in Eq. (16). The resulting expression reads:

$$
\left\langle e^{i \sqrt{2 D_{0}} \boldsymbol{k} \cdot \int_{0}^{\infty} d \boldsymbol{w}(s)\left[H\left(t_{1}-s\right) G\left(t_{1}-s\right)-H\left(t_{2}-s\right) G\left(t_{2}-s\right)\right]}\right\rangle
$$

where we denoted the Heaviside step function by $H(x)$. We have already removed the transient term proportional to the initial condition because we are interested in the asymptotic behaviour of the above Lagrangian correlation function. By means of lengthy integration over the Gaussian measure of the Wiener processes, we get that the foregoing mean value is equivalent to:

$$
e^{-D_{0}\|\boldsymbol{k}\|^{2} \int_{0}^{\infty} d s\left[H\left(t_{1}-s\right) G\left(t_{1}-s\right)-H\left(t_{2}-s\right) G\left(t_{2}-s\right)\right]^{2}}
$$

As detailed in Appendix B, the exponent of (18) can be simplified exploiting the relationship between $G$ and $K_{1}$ - see the rightmost side of Eq. (16) - and the explicit expression for $K_{1}$ 
given by Eq. (7). The results reported in Appendix B lead to the expression for $G$

$$
\begin{aligned}
G(t) & =\frac{1}{2 i A_{1} A_{2} \Im A_{2} \mathrm{St}}\left[2 i \Im A_{2}-A_{2} A_{1} e^{A_{1}^{2} t}+A_{1} A_{2} e^{A_{2}^{2} t}\right] \\
& +\frac{1}{2 i \Im A_{2} \mathrm{St}}\left[\frac{1}{\sqrt{\pi}} \int_{0}^{t} \frac{e^{-A_{1}^{2}(r-t)}-e^{-A_{2}^{2}(r-t)}}{\sqrt{r}} d r\right] \\
& =\frac{1}{A_{1} A_{2} \mathrm{St}}-\frac{1}{2 i \Im A_{2} \mathrm{St}}\left[e^{A_{1}^{2} t}-e^{A_{2}^{2} t}-\frac{1}{\sqrt{\pi}} \int_{0}^{t} \frac{e^{A_{1}^{2} r}-e^{A_{2}^{2} r}}{\sqrt{r+t}} d r\right]
\end{aligned}
$$

from which the eddy diffusivity from Taylor's formula can be determined once $\check{B}$ is known:

$$
\mathbb{D}=D_{0} \mathbb{I}+\int_{0}^{\infty} d t \int \frac{d \boldsymbol{k}^{d-1}}{(2 \pi)^{d-1}} e^{-D_{0}\|\boldsymbol{k}\|^{2}\left[t+2 \int_{0}^{\infty} d s G(s)(G(s)-G(t+s))\right.} \check{B}_{\check{B}}(\boldsymbol{k},|t|) \boldsymbol{e}_{1} \otimes \boldsymbol{e}_{1}
$$

\section{B. Expansion at the first order in molecular diffusivity}

The expression (20) for the eddy diffusivity is now explicit, and one could use it to already get results by numerical computation. Nonethless,its complexity suggests us to proceed to simplify it. A good starting point is to take into account that the molecular diffusivity $D_{0}$ (in dimensionless units) is typically a small number in applications. Along this line, let us keep the leading order in the small $D_{0}$ expansion $^{30,40}$ :

$$
\begin{aligned}
& \mathbb{D} \simeq D_{0} \mathbb{I}+\int_{0}^{\infty} d t \int \frac{d \boldsymbol{k}^{d-1}}{(2 \pi)^{d-1}} \check{B}(\boldsymbol{k},|t|)\left[1-D_{0}\|\boldsymbol{k}\|^{2} t\right] \boldsymbol{e}_{1} \otimes \boldsymbol{e}_{1} \\
& +2 D_{0} \int \frac{d \boldsymbol{k}^{d-1}}{(2 \pi)^{d-1}}\|\boldsymbol{k}\|^{2} \int_{0}^{\infty} d t \check{B}(\boldsymbol{k},|t|) \int_{0}^{\infty} d s G(s)(G(t+s)-G(s)) \boldsymbol{e}_{1} \otimes \boldsymbol{e}_{1}
\end{aligned}
$$

The last addend in Eq. (21) includes the contribution from the Basset history term. Let us focus our attention on it. For the sake of simplicity, let us consider a single-mode, oscillating velocity correlation function having the (dimensionless) wavenumber $k_{0}=1$, a (dimensionless) exponential correlation time 1 and (dimensionless) frequency $\Omega$ :

$$
\check{B}(\boldsymbol{k},|t|)=(2 \pi)^{d-1} E\left(k_{0}\right) e^{-|t|} \cos (\Omega t)\left[\delta\left(\boldsymbol{k}-\boldsymbol{k}_{0}\right)+\delta\left(\boldsymbol{k}+\boldsymbol{k}_{0}\right)\right]
$$

In Appendix $\mathrm{C}$ we report an method (see also Refs. 24 and 44 for other specific applications) about how to generate a flow field having such properties. An exponentially decaying, oscillating correlation function was observed in some flow models where particles were also endowed with a molecular diffusivity ${ }^{45}$.It therefore embodies a representative, meaningful choice, as it captures both diffusion decay and recirculation structures in a simple way . 
By recalling Eq. (22), the term under consideration becomes

$$
4 D_{0} E\left(k_{0}\right) \Delta_{1}(\mathrm{St}, \beta, \Omega) \boldsymbol{e}_{1} \otimes \boldsymbol{e}_{1}
$$

having defined:

$$
\Delta_{1}(\mathrm{St}, \beta, \Omega)=\int_{0}^{\infty} d t e^{-t} \cos (\Omega t) \int_{0}^{\infty} d s G(s)(G(t+s)-G(s))
$$

The eddy diffusivity follows from (21) and reads:

$$
\begin{aligned}
& \mathbb{D} \simeq D_{0} \mathbb{I}+2 E\left(k_{0}\right) \int_{0}^{\infty} d t e^{-|t|} \cos (\Omega t)\left[1-D_{0} t\right] \boldsymbol{e}_{1} \otimes \boldsymbol{e}_{1}+4 D_{0} E\left(k_{0}\right) \Delta_{1}(\mathrm{St}, \beta, \Omega) \boldsymbol{e}_{1} \otimes \boldsymbol{e}_{1} \\
& =D_{0} \mathbb{I}+\left\{2 E\left(k_{0}\right)\left[\frac{1}{1+\Omega^{2}}-D_{0} \frac{1-\Omega^{2}}{\left(\Omega^{2}+1\right)^{2}}\right]+4 D_{0} E\left(k_{0}\right) \Delta_{1}(\mathrm{St}, \beta, \Omega)\right\} \boldsymbol{e}_{1} \otimes \boldsymbol{e}_{1}
\end{aligned}
$$

The sum of the terms proportional to $D_{0}$ needs be much smaller than $2 E\left(k_{0}\right) /\left(1+\Omega^{2}\right)$, in order to remain in a perturbative regime.

Similarly to the most common simplified versions of Eq. (1), which are the cases of heavy particles with $\beta=0$ or when the history term is neglected ${ }^{17,18}$, for shear flows inertia plays a role only when molecular diffusivity is present. Eqs. (20) and (25) indeed yield the same result as the free tracer when $D_{0}=0^{30,40}$ :

$$
\mathbb{D}_{\text {trac }}=\frac{2 E\left(k_{0}\right)}{1+\Omega^{2}} \boldsymbol{e}_{1} \otimes \boldsymbol{e}_{1}
$$

If $D_{0} \neq 0$, however, Eq.(25) tells us that in the full BBO model (1) the eddy diffusivity

depends on the density ratio $\beta$, unlike the above mentioned simplified models ${ }^{40}$. The function $\Delta_{1}$ represents in fact the contribution of Stokes drag and history force term appearing in Eq. (1) to the interference between carrier flow and molecular diffusion. This interference mechanism had been investigated in Ref. 30 for the tracer case. Such a result is a quantitative effect of the Basset history term.

\section{Analysis of test cases}

Our simplifications allowed us to arrive at Eq. (25) where only time integrations are involved. They can be easily performed by standard methods (see below). As explicit test cases, we are going to consider $\beta=1 / 10,1 / 3,1$, and 3 . 
Let us now show the numerical results of the double integral (24) for the above mentioned values of $\beta$, and compare those with the result of in the absence of Basset history term. In this latter case $\mathrm{G}(\mathrm{t})$ is (see also Eq. (C4) in the Appendix B):

$$
G(t)=1-e^{-\frac{t}{\mathrm{St}}}
$$

and in Eq. (24) one would have:

$$
\int_{0}^{\infty} d s G(s)(G(t+s)-G(s))=\frac{\mathrm{St}}{2}\left(1-e^{-\frac{t}{\mathrm{St}}}\right)
$$

Therefore, by exploiting the expression above one gets:

$$
\Delta_{1}=\frac{\mathrm{St}}{2\left(1+\Omega^{2}\right)}-\frac{\mathrm{St}^{2}(\mathrm{St}+1)}{2\left[1+\mathrm{St}\left(2+\mathrm{St}+\mathrm{St} \Omega^{2}\right)\right]}
$$

To calculate the double integral in Eq. (25) numerically when the Basset term is present, the domain for the variables $(t, s)$ of Eq. (25) was initially restricted to a rectangle $\left[0, T_{1}\right] \times$ $\left[0, T_{2}\right]$. Afterwards, the integral was computed through an automatic global adaptive algorithm provided in a commercial software making sure that the result was sufficiently stable under a certain error threshold upon changes of settings. Computation was consequently accomplished by increasing $T_{1}, T_{2}$ up to convergence and assessing such a convergence with respect to the settings per each choice of those two values. The integral proved overall to be convergent within an error of order $\sim 0.1 \%-1 \%$ when $T_{1}=20$ - that is, increasing this number further did not change the result - and $T_{2}$ was varied over the rather wide range $10^{6}-10^{8}$. Values of the quantity $\Delta_{1}$ from this computation are depicted in Figs. 1-2 for $\Omega=0$ and $\Omega=0.8$ respectively. The latter have been chosen similarly to Ref. 40 as representative values for a purely-decaying and a decaying-oscillating correlation function. A strictly positive correlation function like the case $\Omega=0$ in Fig. 1 implicates that on average the flow is globally in the same direction at any time; it represents a limit case with no recirculation. We thus expect the contribution to diffusion from inertia to be always positive. Conversely, when an oscillating correlation function is present, at a certain time we have points where velocities are in opposite directions, a situation that reveals the presence of vortexes and recirculation. In the latter scenario, the particles can remain trapped and the transport can be reduced (see Fig. 2) ${ }^{30}$. Of course the Stokes response time St of the particles needs to be sufficiently small so that they can actually be sensitive to the recirculation time of the flow structures. 
Note that, no matter what density ratio is considered, all the curves seem to converge to the same asymptote as the Basset-less case when St increases. Given that at high St numbers the Basset force decreases like $\mathrm{St}^{-1 / 2}$, all the cases converge to the heavy-particle scenario, $\beta=0$, where Basset's term is also not present. This fact can be used to test the right behaviour of a numerical solver for the $\mathrm{BBO}$ equations when $\mathrm{St}$ is particularly high. Also, in Table I a list of $\Delta_{1}$ values for the smallest and highest values of $\beta$ herein considered is presented for the two different frequencies $\Omega=0,0.8$, in order to be promptly used as a benchmark for numerical testing. For instance, if one sets $D_{0}=0.2, E\left(k_{0}\right)=1$, St $=0.2, \Omega=$ 0 , and $\beta=3$ in a numerical solver for the BBO equation, by reading Table I and plugging the numbers into Eq. (25), the result is $D_{11}=2.1064$. The sum of the terms proportional to $D_{0}$ in Eq. (25) is -0.0936 , which is sufficiently smaller than 2, thus confirming we are safely in a perturbative regime where our formulas are valid. It is finally important to point out that the integral in Eq (25) could be evaluated via standard techniques at even higher precision than the one reported in the table, where necessary.

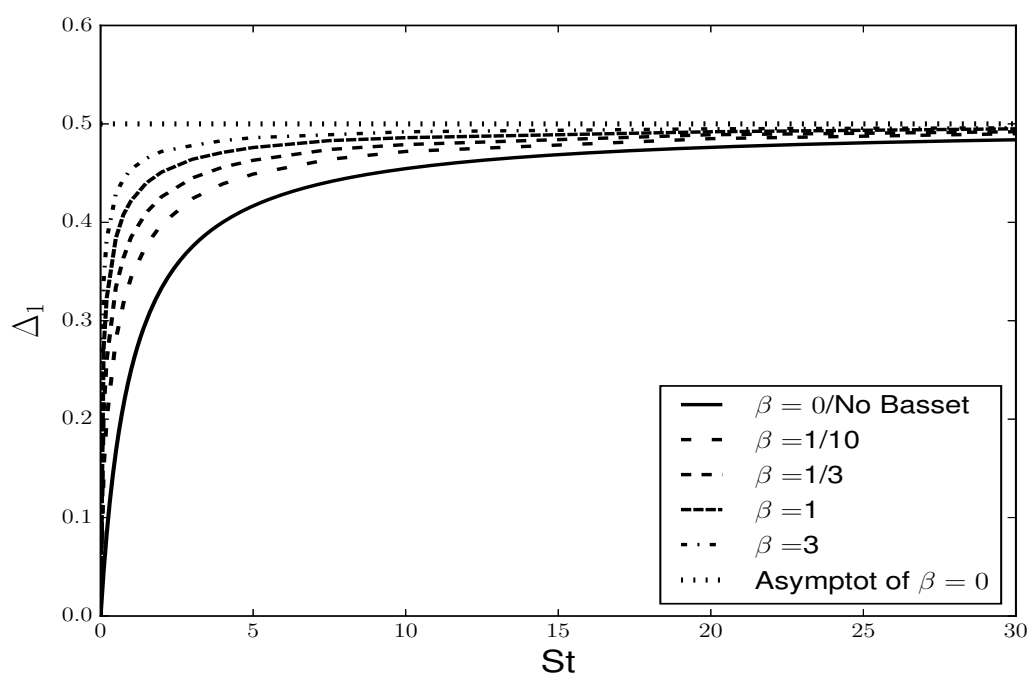

FIG. 1. Values of $\Delta_{1}$ at different Stokes and $\beta$ compared to the asymptote for $\beta=0$ when St $\rightarrow \infty$. This term represent the contribution to diffusion caused by inertia - Stokes drag and Basset force. The asymptote is evidently the same for any $\beta$. Here $\Omega=0$. All the cases converge to the heavyparticle scenario, $\beta=0$, where Basset's term is not present. The monotonic behaviour is due to the absence of the recirculating structures, resulting in the particle inertia always enhancing the long-time diffusion. 

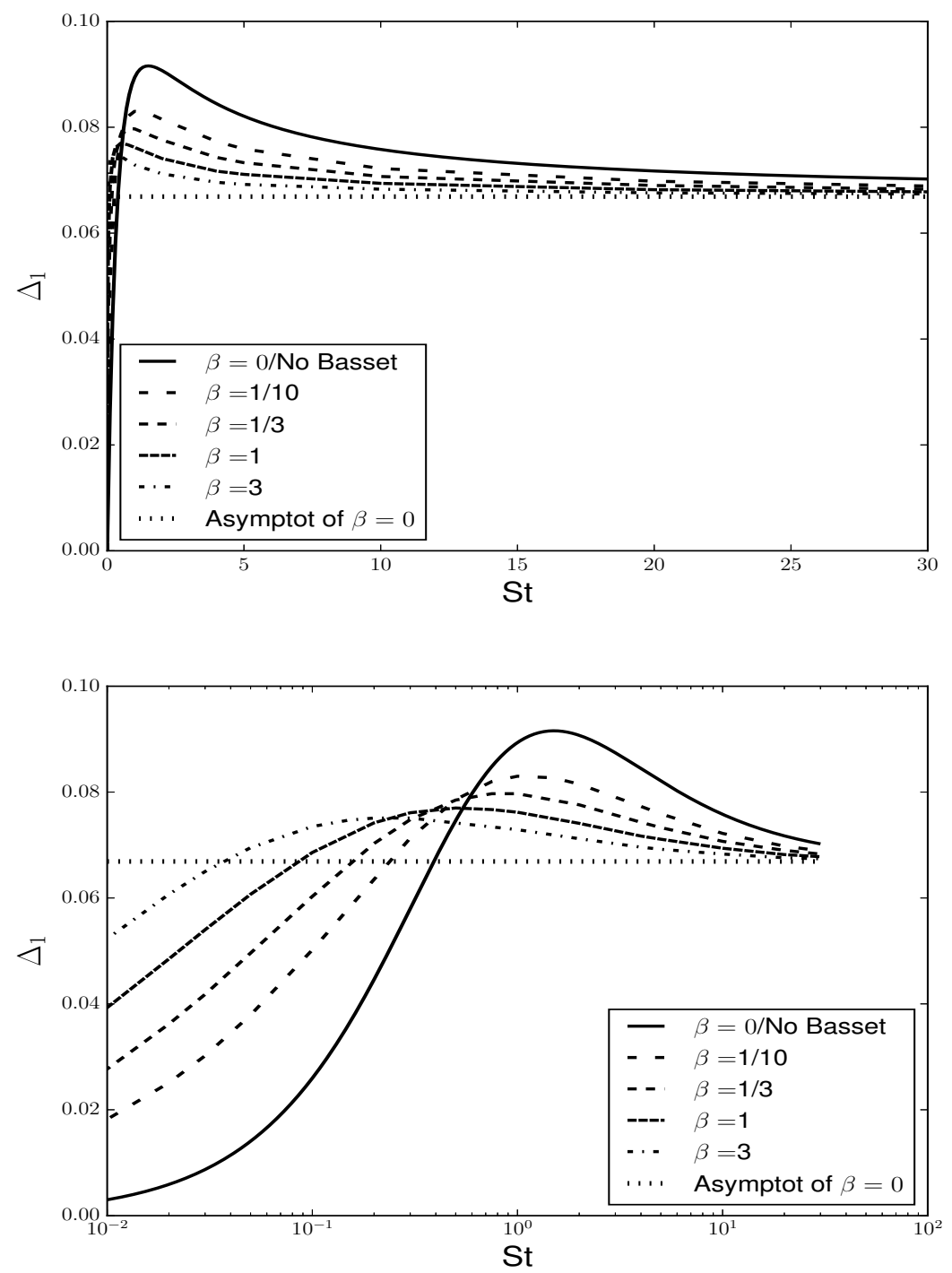

FIG. 2. Values of $\Delta_{1}$, the inertia contribution to the diffusion, for $\Omega=0.8$. Plots are at different Stokes and $\beta$, and are compared to the asymptote of $\beta=0$ when St $\rightarrow \infty$. Upper panel: linear scale. Lower panel: semilogarithmic scale for the St-axis, in order to view the curves better at low St. The presence of an oscillating autocorrelation reveals the existence of recirculation structures. Thus it is possible to have a reduction to the transport when the response time St is small enough for the particle to be sensitive to those structures. 
TABLE I. Values of the inertia contribution $\Delta_{1}$ for the lowest and the highest $\beta$ analyzed, at $\Omega=0$ (upper panel) and 0.8 (lower panel) with respect to Stokes number. With the numerical method and settings used herein, the error amounts to $\sim 0.1 \%-1 \%$, including the round-off.

\begin{tabular}{|c|c|c|c|c|c|c|c|c|c|c|c|c|}
\hline \hline \multicolumn{10}{|c|}{$\Delta_{1} \Omega_{\Omega=0}$} \\
\hline St & 0.1 & 0.2 & 0.3 & 0.5 & 0.75 & 1 & 2 & 4 & 5 & 10 & 20 & 30 \\
\hline$\beta=0.1$ & 0.149 & 0.200 & 0.235 & 0.281 & 0.318 & 0.344 & 0.398 & 0.439 & 0.449 & 0.472 & 0.485 & 0.490 \\
\hline$\beta=3$ & 0.340 & 0.383 & 0.405 & 0.429 & 0.444 & 0.454 & 0.472 & 0.483 & 0.486 & 0.492 & 0.495 & 0.496 \\
\hline \hline
\end{tabular}

\begin{tabular}{|c|c|c|c|c|c|c|c|c|c|c|c|c|}
\hline \hline \multicolumn{10}{|c|}{$\left.\Delta_{1}\right|_{\Omega=0.8}$} \\
\hline St & 0.01 & 0.05 & 0.1 & 0.2 & 0.3 & 0.5 & 1 & 2 & 5 & 10 & 20 & 30 \\
\hline$\beta=0.1$ & 0.0182 & 0.0379 & 0.0502 & 0.0639 & 0.0713 & 0.0830 & 0.0786 & 0.0815 & 0.0759 & 0.0722 & 0.0698 & 0.0689 \\
\hline$\beta=3$ & 0.0523 & 0.0695 & 0.0735 & 0.0751 & 0.0752 & 0.0745 & 0.0729 & 0.0712 & 0.0692 & 0.0683 & 0.0676 & 0.0674 \\
\hline \hline
\end{tabular}

\section{RESULTS FOR THE MAXEY-RILEY EQUATIONS}

Let us now tackle the same problem of determining the eddy diffusivity for another widely used model for the dynamics of inertial particles. The model we are going to consider is described in terms of the full, dimensionless Maxey-Riley equations:

$$
\begin{aligned}
\frac{\mathrm{d} \boldsymbol{v}}{\mathrm{d} t}(t) & =\frac{\boldsymbol{u}(\boldsymbol{\xi}(t), t)-\boldsymbol{v}(t)+\frac{1}{6} r_{p}^{2} \nabla^{2} \boldsymbol{u}(\boldsymbol{\xi}(t), t)}{\mathrm{St}}+\beta \frac{\mathrm{D} \boldsymbol{u}(\boldsymbol{\xi}(t), t)}{\mathrm{D} t}+\frac{\beta}{30} r_{p}^{2} \frac{\mathrm{d}}{\mathrm{d} t} \nabla^{2} \boldsymbol{u}(\boldsymbol{\xi}(t), t) \\
& +\sqrt{\frac{3 \beta}{\pi \mathrm{St}}} \int_{0}^{t} \frac{\mathrm{d} s}{\sqrt{t-s}} \frac{\mathrm{d}}{\mathrm{d} s}\left(\boldsymbol{u}(\boldsymbol{\xi}(s), s)-\boldsymbol{v}(s)+\frac{1}{6} r_{p}^{2} \nabla^{2} \boldsymbol{u}(\boldsymbol{\xi}(s), s)\right)+\frac{\sqrt{2 D_{0}}}{\mathrm{St}} \boldsymbol{\eta}(t)
\end{aligned}
$$

with $\frac{\mathrm{D}}{\mathrm{D} t}=\partial_{t}+\boldsymbol{u} \cdot \boldsymbol{\nabla}$.

Maxey and Riley derived this model in quite a rigorous manner (see Ref. 14), and can be considered as a refinement of the prior version in Eq. (1) In fact, it takes into account higher order corrections with respect to the particle ratio $r_{p} / L \ll 1$ and the dimensionless group St $\beta \sim r_{p}^{2} /(T \nu) \ll 1$, with $L$ and $T$ being some characteristic length scale and time scale of the fluid field, respectively. In particular, note that the pressure gradient causing the added mass effect is now proportional to the acceleration of the fluid particle, rather than the inertial particle like in Eq. (1). Besides, the higher order corrections in $r_{p} / L$ constitute the so-called Faxén drag, representing the interaction between the particle volume and the 
curvature of the underlying flow lines.

Along the directions $n \neq 1$ the flow velocity is zero and Eq. (29) coincides with Eq. (1). It then follows that the components $n \neq 1$ of the trajectories are still given by Eq. (16). Taking into account the initial condition $\boldsymbol{v}(0)=\boldsymbol{u}(\boldsymbol{\xi}(0), 0)$, the term depending on it decays asymptotically with respect to time, and we can set it to 0 from the very beginning by putting $\boldsymbol{v}(0)=\boldsymbol{u}(\boldsymbol{\xi}(0), 0)=0$. This will not cause loss of generality, because we are interested in asymptotic properties of the transport problem.

As detailed in Ref. 22, a generalized Taylor's formula for the model (29) does not involve the sole flow velocity correlations. New terms do appear and the resulting eddy diffusivity reads:

$$
\begin{aligned}
\mathrm{D}=D_{0} \mathbb{1} & +\int_{0}^{\infty} \mathrm{d} t\left\langle\left[\boldsymbol{u}(\boldsymbol{\xi}(t), t)+\beta \mathrm{St} \frac{\mathrm{D} \boldsymbol{u}}{\mathrm{D} t}(\boldsymbol{\xi}(t), t)+\frac{1}{6} r_{p}^{2} \nabla^{2} \boldsymbol{u}(\boldsymbol{\xi}(t), t)\right]\right. \\
& \left.\otimes\left[\boldsymbol{u}(\boldsymbol{\xi}(0), 0)+\beta \mathrm{St} \frac{\mathrm{D} \boldsymbol{u}}{\mathrm{D} t}(\boldsymbol{\xi}(0), 0)+\frac{1}{6} r_{p}^{2} \nabla^{2} \boldsymbol{u}(\boldsymbol{\xi}(0), 0)\right]\right\rangle
\end{aligned}
$$

\section{A. Eddy diffusivity for shear flows: the full Maxey-Riley model}

We can now work to simplify the expression 30 by restricting ourselves to shear flows and availing ourselves of their properties. The starting point is to note that, in such flows, $\frac{\mathrm{D} \boldsymbol{u}}{\mathrm{D} t}(\boldsymbol{x}, t)=\partial_{t} \boldsymbol{u}(\boldsymbol{x}, t)$ evaluated in $\boldsymbol{x}=\boldsymbol{\xi}(t)$. Moreover, we can exploit both the stationarity of the Lagrangian correlation function and the linearity of the derivation operator:

$$
\begin{gathered}
\left\langle\boldsymbol{u}(\boldsymbol{\xi}(t), t) \otimes \frac{\partial \boldsymbol{u}}{\partial t}(\boldsymbol{\xi}(0), 0)\right\rangle=-\left\langle\frac{\partial \boldsymbol{u}}{\partial t}(\boldsymbol{\xi}(t), t) \otimes \boldsymbol{u}(\boldsymbol{\xi}(0), 0)\right\rangle \\
\left\langle\nabla^{2} \boldsymbol{u}(\boldsymbol{\xi}(t), t) \otimes \frac{\partial \boldsymbol{u}}{\partial t}(\boldsymbol{\xi}(0), 0)\right\rangle=-\left\langle\frac{\partial \boldsymbol{u}}{\partial t}(\boldsymbol{\xi}(t), t) \otimes \nabla^{2} \boldsymbol{u}(\boldsymbol{\xi}(0), 0)\right\rangle
\end{gathered}
$$

We also have that for a general correlation function $f: \mathbb{R} \rightarrow \mathbb{R}$

$$
\begin{aligned}
\nabla_{r_{1}}^{2} \nabla_{r_{2}}^{2} f\left(\left|\boldsymbol{r}_{1}-\boldsymbol{r}_{2}\right|\right) & =\nabla_{r_{1}}^{4} f\left(\left|\boldsymbol{r}_{1}-\boldsymbol{r}_{2}\right|\right) \\
\frac{\partial}{\partial t_{1}} \frac{\partial}{\partial t_{2}} f\left(\left|t_{1}-t_{2}\right|\right) & =-\frac{\partial^{2}}{\partial t_{1}^{2}} f\left(\left|t_{1}-t_{2}\right|\right)
\end{aligned}
$$

Such a property follows from the fact that the Laplace operator does not change the spatial parity of the homogeneous correlation function. Its time derivative, however, is an odd 
function of time, as the time correlation function is stationary and, as a consequence, an even one.

As a result of all the above considerations, the following expression is obtained:

$$
\begin{aligned}
\mathbb{D}=D_{0} \mathbb{1} & +\int_{0}^{\infty} \mathrm{d} t\left\langle\left[\boldsymbol{u}(\boldsymbol{\xi}(t), t)+\beta \mathrm{St} \frac{\partial \boldsymbol{u}}{\partial t}(\boldsymbol{\xi}(t), t)+\frac{1}{6} r_{p}^{2} \nabla^{2} \boldsymbol{u}(\boldsymbol{\xi}(t), t)\right]\right. \\
& \left.\otimes\left[\boldsymbol{u}(\boldsymbol{\xi}(0), 0)+\beta \mathrm{St} \frac{\partial \boldsymbol{u}}{\partial t}(\boldsymbol{\xi}(0), 0)+\boldsymbol{u}(\boldsymbol{\xi}(0), 0)\right]\right\rangle
\end{aligned}
$$

The products can be expanded and recast to get:

$$
\begin{aligned}
\mathbb{D}=D_{0} \mathbb{1} & +\int_{0}^{\infty} \mathrm{d} t[\langle\boldsymbol{u}(\boldsymbol{\xi}(t), t) \otimes \boldsymbol{u}(\boldsymbol{\xi}(0), 0)\rangle \\
& +\beta^{2} \mathrm{St}^{2}\left\langle\frac{\partial \boldsymbol{u}}{\partial t}(\boldsymbol{\xi}(t), t) \otimes \frac{\partial \boldsymbol{u}}{\partial t}(\boldsymbol{\xi}(0), 0)\right\rangle \\
& +\left\langle\boldsymbol{u}(\boldsymbol{\xi}(t), t) \otimes \frac{1}{6} r_{p}^{2} \nabla^{2} \boldsymbol{u}(\boldsymbol{\xi}(0), 0)+\boldsymbol{u}(\boldsymbol{\xi}(0), 0) \otimes \frac{1}{6} r_{p}^{2} \nabla^{2} \boldsymbol{u}(\boldsymbol{\xi}(t), t)\right\rangle \\
& \left.+\left\langle\frac{1}{6} r_{p}^{2} \nabla^{2} \boldsymbol{u}(\boldsymbol{\xi}(t), t) \otimes \frac{1}{6} r_{p}^{2} \nabla^{2} \boldsymbol{u}(\boldsymbol{\xi}(0), 0)\right\rangle\right]
\end{aligned}
$$

and, after trivial algebraic manipulation, the latter in turns corresponds to:

$$
\begin{aligned}
& =\int_{0}^{\infty} \mathrm{d} t\left[\langle\boldsymbol{u}(\boldsymbol{\xi}(t), t) \otimes \boldsymbol{u}(\boldsymbol{\xi}(0), 0)\rangle-\beta^{2} \mathrm{St}^{2} \frac{\partial^{2}}{\partial t^{2}}\langle\boldsymbol{u}(\boldsymbol{\xi}(t), t) \otimes \boldsymbol{u}(\boldsymbol{\xi}(0), 0)\rangle\right. \\
& \left.+\left\langle\nabla^{2} \boldsymbol{u}(\boldsymbol{\xi}(t), t) \otimes \frac{1}{3} r_{p}^{2} \boldsymbol{u}(\boldsymbol{\xi}(0), 0)\right\rangle+\left\langle\frac{1}{36} r_{p}^{4} \nabla^{4} \boldsymbol{u}(\boldsymbol{\xi}(t), t) \otimes \boldsymbol{u}(\boldsymbol{\xi}(0), 0)\right\rangle\right]
\end{aligned}
$$

This expression involves four different terms which we are going to evaluate step-by-step. This will be done first in the general form and afterwards specializing in the monochromatic flow field (22).

The first addend in the first line of Eq. (35) has been already calculated in Eq. (15), as the trajectories along the directions $n \neq 1$ are the same as in Eq. (16).

The second addend of the first line originated from the autocorrelation along the inertial particle trajectory of the fluid particle acceleration, which was in turn an estimation of the pressure gradient around the inertial particle ${ }^{14}$. This addend can be promptly computed: 


$$
\begin{aligned}
& \beta^{2} \mathrm{St}^{2} \frac{\partial^{2}}{\partial t^{2}}\langle\boldsymbol{u}(\boldsymbol{\xi}(t), t) \otimes \boldsymbol{u}(\boldsymbol{\xi}(0), 0)\rangle \\
= & \beta^{2} \mathrm{St}^{2} \frac{\partial^{2}}{\partial t^{2}}\left[\int \frac{d \boldsymbol{k}^{d-1}}{(2 \pi)^{d-1}}\left\langle e^{i \boldsymbol{k} \cdot(\boldsymbol{\xi}(t)-\boldsymbol{\xi}(0))}\right\rangle \check{B}(\boldsymbol{k},|t|) \boldsymbol{e}_{1} \otimes \boldsymbol{e}_{1}\right] \\
= & \beta^{2} \mathrm{St}^{2} \int \frac{d \boldsymbol{k}^{d-1}}{(2 \pi)^{d-1}} e^{-D_{0}\|\boldsymbol{k}\|^{2}\left[t+2 \int_{0}^{\infty} d s G(s)(G(s)-G(t+s))\right]} \frac{\partial^{2}}{\partial t^{2}} \check{B}(\boldsymbol{k},|t|) \boldsymbol{e}_{1} \otimes \boldsymbol{e}_{1}
\end{aligned}
$$

Finally, we are only left with the two terms in the second line of Eq. (35) depending on the particle radius. They respectively represent the crosscorrelation between Faxén drag and flow velocity and the autocorrelation of the Faxén drag itself. Those two addends embody the fact that, when letting a particle have a non-null volume, a spatial correlation between noncontiguous point arises. In plain works, what matters is no more just the autocorrelation along each point trajectory like in Taylor's 1922 formula, but the correlation between the points that the volume of each particle occupied during the motion. By taking into account Eq. (15):

$$
\begin{aligned}
& \left\langle\nabla^{2} \boldsymbol{u}(\boldsymbol{\xi}(t), t) \otimes \frac{1}{3} r_{p}^{2} \boldsymbol{u}(\boldsymbol{\xi}(0), 0)\right\rangle+\left\langle\frac{1}{36} r_{p}^{4} \nabla^{4} \boldsymbol{u}(\boldsymbol{\xi}(t), t) \otimes \boldsymbol{u}(\boldsymbol{\xi}(0), 0)\right\rangle \\
= & \int \frac{d \boldsymbol{k}^{d-1}}{(2 \pi)^{d-1}} \int_{0}^{\infty} \mathrm{d} t e^{-D_{0}\|\boldsymbol{k}\|^{2}\left[t+2 \int_{0}^{\infty} d s G(s)(G(s)-G(t+s))\right]} \\
& \times\left[\frac{1}{3} r_{p}^{2}\|\boldsymbol{k}\|^{2}+\frac{1}{36} r_{p}^{4}\|\boldsymbol{k}\|^{4}\right] \check{B}(\boldsymbol{k},|t|) \boldsymbol{e}_{1} \otimes \boldsymbol{e}_{1}
\end{aligned}
$$

Now let us again leverage the shear flow hypothesis in Eqs. (36) and (37). If we plug the correlation (22) into Eq. (36), we arrive at:

$$
\begin{aligned}
& \beta^{2} \mathrm{St}^{2} \frac{\partial^{2}}{\partial t^{2}}\langle\boldsymbol{u}(\boldsymbol{\xi}(t), t) \otimes \boldsymbol{u}(\boldsymbol{\xi}(0), 0)\rangle \\
= & 2 \beta^{2} \mathrm{St}^{2} e^{-D_{0}\left\|\boldsymbol{k}_{0}\right\|^{2}}\left[t+2 \int_{0}^{\infty} d s G(s)(G(s)-G(t+s))\right] E\left(k_{0}\right) \\
\times & {\left[-2 \delta(t)+\left(1-\Omega^{2}\right) e^{-|t|} \cos (\Omega t)+2 \Omega e^{-|t|} \sin (\Omega|t|)\right] \boldsymbol{e}_{1} \otimes \boldsymbol{e}_{1} }
\end{aligned}
$$

We need to integrate the above expression with respect to time in order to be able to plug 
it into Eq. (35) later:

$$
\begin{aligned}
& \beta^{2} \mathrm{St}^{2} \int_{0}^{\infty} \mathrm{d} t \frac{\partial^{2}}{\partial t^{2}}\langle\boldsymbol{u}(\boldsymbol{\xi}(t), t) \otimes \boldsymbol{u}(\boldsymbol{\xi}(0), 0)\rangle \\
= & 2 \beta^{2} \mathrm{St}^{2} \int_{0}^{\infty} \mathrm{d} t e^{-D_{0}\left\|\boldsymbol{k}_{0}\right\|^{2}\left[t+2 \int_{0}^{\infty} d s G(s)(G(s)-G(t+s))\right]} \\
\times & E\left(k_{0}\right)\left[-2 \delta(t)+\left(1-\Omega^{2}\right) e^{-|t|} \cos (\Omega t)+2 \Omega e^{-|t|} \sin (\Omega|t|)\right] \boldsymbol{e}_{1} \otimes \boldsymbol{e}_{1}
\end{aligned}
$$

By virtue of the formal identity $\int_{0}^{\infty} d t \delta(t)=1 / 2$, we promptly see that when $D_{0}=0$, this integral equals 0 . In this case, the difference between the eddy diffusivities in the MaxeyRiley and the Basset-Boussinesq-Oseen models lies only in the terms originating from the Faxén friction; by considering the flow in Eq.(22), Eq. (37) becomes:

$$
\begin{aligned}
& \left\langle\nabla^{2} \boldsymbol{u}(\boldsymbol{\xi}(t), t) \otimes \frac{1}{3} r_{p}^{2} \boldsymbol{u}(\boldsymbol{\xi}(0), 0)\right\rangle+\left\langle\frac{1}{36} r_{p}^{4} \nabla^{4} \boldsymbol{u}(\boldsymbol{\xi}(t), t) \otimes \boldsymbol{u}(\boldsymbol{\xi}(0), 0)\right\rangle \\
= & 2 e^{-D_{0}\left[t+2 \int_{0}^{\infty} d s G(s)(G(s)-G(t+s))\right]}\left(\frac{1}{3} r_{p}^{2}+\frac{1}{36} r_{p}^{4}\right) E\left(k_{0}\right) e^{-|t|} \cos (\Omega t) \boldsymbol{e}_{1} \otimes \boldsymbol{e}_{1}
\end{aligned}
$$

Again $r_{p}$ has now been nondimesionalized with respect to the charcteristic flow wavelength $2 \pi / k_{0}=2 \pi$. Taking into account the time integral in Eq. (35) and Eq. (26), when $D_{0}=0$ the eddy diffusivity for a stochastic shear flow is:

$$
\mathrm{D}=\frac{2 E\left(k_{0}\right)}{1+\Omega^{2}}\left(1+\frac{1}{3} r_{p}^{2}+\frac{1}{36} r_{p}^{4}\right) \boldsymbol{e}_{1} \otimes \boldsymbol{e}_{1}
$$

It is important to recall that for this model to hold true, it must be $r_{p} \ll 1$. Faxén drag always creates a transport enhancement, albeit of small magnitude.

\section{B. Expansion at the first order in the molecular diffusivity}

If $D_{0} \neq 0$, we can again carry out a first-order expansion with respect to the dimensionless molecular diffusivity $D_{0}$, due to the same reason we have stated in Sec. IIB. The expansion of Eq. (36) is:

$$
\begin{aligned}
& \beta^{2} \mathrm{St}^{2} \frac{\partial^{2}}{\partial t^{2}}\langle\boldsymbol{u}(\boldsymbol{\xi}(t), t) \otimes \boldsymbol{u}(\boldsymbol{\xi}(0), 0)\rangle \\
\simeq & \beta^{2} \mathrm{St}^{2} \int \frac{d \boldsymbol{k}^{d-1}}{(2 \pi)^{d-1}}\left\{1-D_{0}\|\boldsymbol{k}\|^{2}\left[t+2 \int_{0}^{\infty} d s G(s)(G(s)-G(t+s))\right]\right\} \frac{\partial^{2}}{\partial t^{2}} \check{B}(\boldsymbol{k},|t|) \boldsymbol{e}_{1} \otimes \boldsymbol{e}_{1}
\end{aligned}
$$


and, evaluating it for the correlation (22):

$$
\begin{aligned}
& \int_{0}^{\infty} \mathrm{d} t \beta^{2} \mathrm{St}^{2} \frac{\partial^{2}}{\partial t^{2}}\langle\boldsymbol{u}(\boldsymbol{\xi}(t), t) \otimes \boldsymbol{u}(\boldsymbol{\xi}(0), 0)\rangle \\
\simeq & 2 \beta^{2} \mathrm{St}^{2} \int_{0}^{\infty} \mathrm{d} t\left\{1-D_{0}\left[t+2 \int_{0}^{\infty} d s G(s)(G(s)-G(t+s))\right]\right\} \\
\times & E\left(k_{0}\right)\left[-2 \delta(t)+\left(1-\Omega^{2}\right) e^{-|t|} \cos (\Omega t)+2 \Omega e^{-|t|} \sin (\Omega|t|)\right] \boldsymbol{e}_{1} \otimes \boldsymbol{e}_{1} \\
= & 2 \beta^{2} \mathrm{St}^{2} \int_{0}^{\infty} \mathrm{d} t\left\{D_{0}\left[t+2 \int_{0}^{\infty} d s G(s)(G(t+s)-G(s))\right]\right\} \\
\times & E\left(k_{0}\right)\left[\left(1-\Omega^{2}\right) e^{-|t|} \cos (\Omega t)+2 \Omega e^{-|t|} \sin (\Omega|t|)\right] \boldsymbol{e}_{1} \otimes \boldsymbol{e}_{1} \\
= & 2 D_{0} \beta^{2} \mathrm{St}^{2} E\left(k_{0}\right)\left[-1+2\left(1-\Omega^{2}\right) \Delta_{1}(\mathrm{St}, \beta, \Omega)+4 \Omega \Delta_{2}(\mathrm{St}, \beta, \Omega)\right] \boldsymbol{e}_{1} \otimes \boldsymbol{e}_{1} .
\end{aligned}
$$

In the above expression the following function was introduced to ease the notation:

$$
\Delta_{2}(\mathrm{St}, \beta, \Omega)=\int_{0}^{\infty} d t e^{-t} \sin (\Omega t) \int_{0}^{\infty} d s G(s)(G(t+s)-G(s))
$$

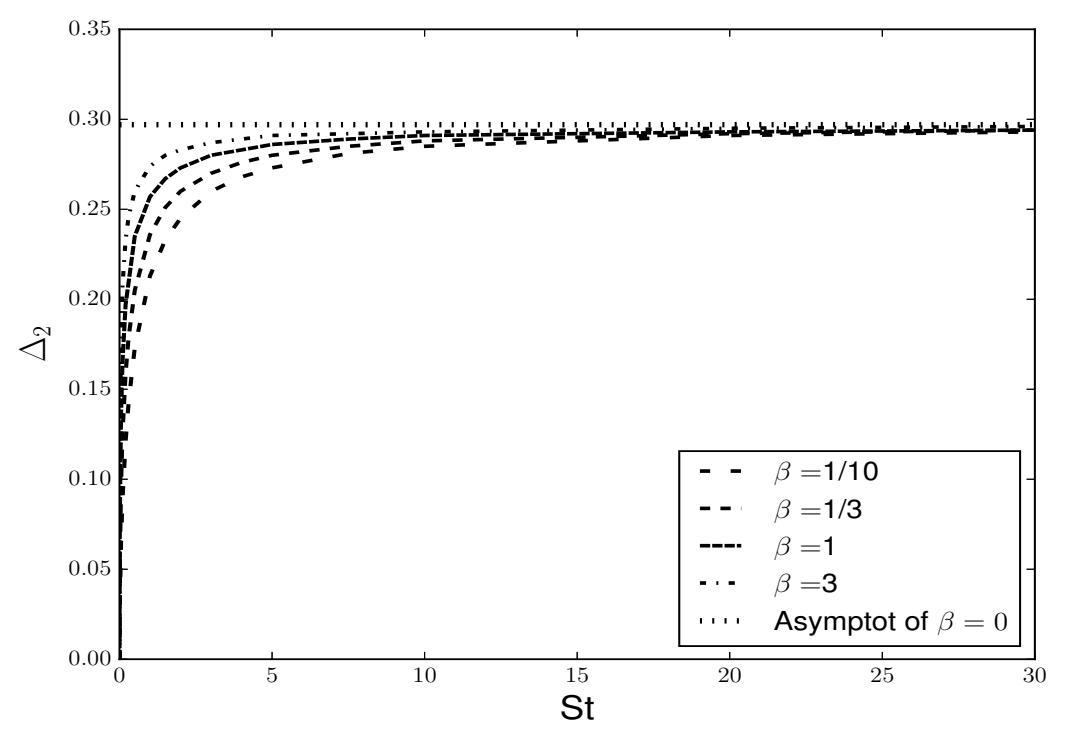

FIG. 3. Values of $\Delta_{2}$ at different Stokes and $\beta$ compared to the asymptote when $\mathrm{St} \rightarrow \infty$. The asymptote is evidently the same for any $\beta$. Here $\Omega=0.8$. This term is proportional to the diffusion reduction caused by the fluid acceleration autocorrelation that is out of phase with respect to the flow velocity autocorrelation along each inertial particle trajectory.

The contribution to the eddy diffusivity in Eq.(43) comes from the autocorrelation of the fluid particle acceleration as already said, and it depends on both $\Delta_{1}$ and $\Delta_{2}$, the former 
being proportional to the autocorrelation of the velocity. The expression (44) encapsulates the contribution from the fluid acceleration autocorrelation that is out-of-phase with respect to the velocity. This can be easily noticed by contrasting Eq. (44) to Eq. (24). Evidently it is not zero only when $\Omega \neq 0$. From a physical point of view, it means that the advective time derivative, which appears in Eq. (29) in contrast to the Lagrangian time derivative of Eq. (1), creates a difference in diffusion where recirculation is strong ${ }^{45}$. From Fig. 3 it is clear that it always provides a reduction to the diffusion, given the global negative sign appearing in Eq. (35) in front of the term depicted in Eq. (43). From the figure, it is also immediately clear that the asymptote again is independent of $\beta$. One could therefore exploit Eq. (27) and calculate:

$$
\Delta_{2}(\mathrm{St}, \beta=0, \Omega)=\frac{(\Omega+2 \mathrm{St} \Omega) \mathrm{St}}{2\left(1+\Omega^{2}\right)\left[1+\mathrm{St}\left(1+\mathrm{St}+\mathrm{St} \Omega^{2}\right)\right]}
$$

Hence, the asymptote valid for any $\beta$ is:

$$
\lim _{\mathrm{St} \rightarrow \infty} \Delta_{2}(\mathrm{St}, \beta=0, \Omega)=\frac{\Omega}{\left(1+\Omega^{2}\right)^{2}}
$$

which equals $\simeq 0.297$ when $\Omega=0.8$. Data in Table II shows the numerical computation of the integral (44) is compatible with this asymptote. However, it should be borne in mind that the last line of (43) altogether is proportional to $(\beta \mathrm{St})^{2}$ and its absolute value also increases with respect to $\Omega$. Thus, the higher $\Omega$, the lower product $D_{0} \operatorname{St} \beta$ is allowed in order to remain in a perturbation regime.

TABLE II. Values of $\Delta_{2}$ for the lowest and the highest $\beta$ analysed at $\Omega=0.8$ with respect to Stokes number. With the numerical method and settings used herein, the error amounts to $\sim 0.1 \%-1 \%$, including the round-off.

\begin{tabular}{|c|c|c|c|c|c|c|c|c|c|c|c|c|}
\hline \hline \multicolumn{10}{|c|}{$\Delta_{2} \mid \Omega=0.8$} \\
\hline St & 0.01 & 0.05 & 0.1 & 0.2 & 0.3 & 0.5 & 1 & 2 & 5 & 10 & 20 & 30 \\
\hline$\beta=0.1$ & 0.0301 & 0.0648 & 0.0893 & 0.121 & 0.143 & 0.173 & 0.213 & 0.245 & 0.273 & 0.285 & 0.291 & 0.293 \\
\hline$\beta=3$ & 0.112 & 0.179 & 0.208 & 0.233 & 0.24 & 0.260 & 0.274 & 0.283 & 0.291 & 0.293 & 0.295 & 0.296 \\
\hline \hline
\end{tabular}


As for the terms involving the Faxén force, from Eq. (40) one has:

$$
\begin{aligned}
& \left\langle\nabla^{2} \boldsymbol{u}(\boldsymbol{\xi}(t), t) \otimes \frac{1}{3} r_{p}^{2} \boldsymbol{u}(\boldsymbol{\xi}(0), 0)\right\rangle+\left\langle\frac{1}{36} r_{p}^{4} \nabla^{4} \boldsymbol{u}(\boldsymbol{\xi}(t), t) \otimes \boldsymbol{u}(\boldsymbol{\xi}(0), 0)\right\rangle \\
\simeq & 2\left\{1-D_{0}\left[t+2 \int_{0}^{\infty} d s G(s)(G(s)-G(t+s))\right]\right\}\left(\frac{1}{3} r_{p}^{2}+\frac{1}{36} r_{p}^{4}\right) E\left(k_{0}\right) e^{-|t|} \cos (\Omega t) \boldsymbol{e}_{1} \otimes \boldsymbol{e}_{1}
\end{aligned}
$$

Its time integral is:

$$
\begin{aligned}
& \int_{0}^{\infty} \mathrm{d} t\left\{\left\langle\nabla^{2} \boldsymbol{u}(\boldsymbol{\xi}(t), t) \otimes \frac{1}{3} r_{p}^{2} \boldsymbol{u}(\boldsymbol{\xi}(0), 0)\right\rangle+\left\langle\frac{1}{36} r_{p}^{4} \nabla^{4} \boldsymbol{u}(\boldsymbol{\xi}(t), t) \otimes \boldsymbol{u}(\boldsymbol{\xi}(0), 0)\right\rangle\right\} \\
\simeq & 2\left\{\frac{1}{1+\Omega^{2}}-D_{0} \frac{1-\Omega^{2}}{\left(\Omega^{2}+1\right)^{2}}+2 D_{0} \Delta_{1}(\mathrm{St}, \beta, \Omega)\right\}\left(\frac{1}{3} r_{p}^{2}+\frac{1}{36} r_{p}^{4}\right) E\left(k_{0}\right) \boldsymbol{e}_{1} \otimes \boldsymbol{e}_{1}
\end{aligned}
$$

Looking at the full Taylor formula (35) and plugging in all the contribution from Eqs. (25), (43) and (46), we get the complete expression at the first order in $D_{0}$ for the eddy diffusivity:

$$
\begin{aligned}
\mathbb{D} & \simeq D_{0} \mathbb{I}+\frac{2 E\left(k_{0}\right)}{1+\Omega^{2}} \boldsymbol{e}_{1} \otimes \boldsymbol{e}_{1}+2 D_{0} E\left(k_{0}\right)\left\{\left[-\frac{1-\Omega^{2}}{\left(\Omega^{2}+1\right)^{2}}+2 \Delta_{1}(\mathrm{St}, \beta, \Omega)\right]\left(1+\frac{1}{3} r_{p}^{2}+\frac{1}{36} r_{p}^{4}\right)\right. \\
& \left.-\beta^{2} \mathrm{St}^{2}\left[-1+2\left(1-\Omega^{2}\right) \Delta_{1}(\mathrm{St}, \beta, \Omega)+4 \Omega \Delta_{2}(\mathrm{St}, \beta, \Omega)\right]\right\} \boldsymbol{e}_{1} \otimes \boldsymbol{e}_{1}
\end{aligned}
$$

Of course, for the approximation to be valid, the term within the curly brackets must be far smaller than $1 /\left[D_{0}\left(1+\Omega^{2}\right)\right]$.

In the same way as Eq. (25), Eq. (47) along with Tables I-II can be used to calculate the component $D_{11}$ of the eddy diffusivity tensor. By way of example, let us suppose to set $D_{0}=0.2, r_{p}=0.1, E\left(k_{0}\right)=1$, St $=0.2, \Omega=0$, and $\beta=3$ in a numerical solver for the MR equation. By reading the Tables I-II and plugging the numbers into Eq. (47), the result is $D_{11}=1.9398$. The result fulfills the perturbation hypothesis as the part proportional to $D_{0}$ of the expansion (47) - the one between curly brackets - is 0.0301, thus much smaller than 1. It can be therefore used as a benchmark to assess the validity of a solver of the ruling equations (29), including not only the computationally demanding Basset history term but also the convective derivative contribution and the Faxén drag force.

\section{CONCLUSION}

In this work we have determined the eddy diffusivity for a wide class of inertial particles in shear flows for both any Stokes number and any particle density. This was done analytically 
both for the Basset-Boussinesq-Oseen model, where the history force makes the biggest difference in the asymptotic diffusion dynamics, and the Maxey-Riley equation, where both the advective time derivative and the Faxén drag play a further role. The exact results we obtained for the eddy diffusivity gave us insight about how the several terms affect asymptotic transport regime. Although only for shear flows, these results represent a significant, analytical benchmark for numerical solvers and algorithms for either BBO or MR equations when one has the molecular Brownian motion, a stochastic carrier flow and is interested in testing the long-time accuracy . In such equations, those terms plus the history force and the possible further higher order corrections with respect to the Stokes number have a mutual influence which the scenario rather complex. The significance of these results lies in the lack of exact quantitative results for $\mathrm{BBO}$ and $\mathrm{MR}$ models having all the above mentioned features accounted for. Within this context, tables and graphes to substantiate numerical testing have been included too.

\section{ACKNOWLEDGMENTS}

I wish to thank the AtMath project at University of Helsinki for their financial support.

\section{Appendix A: Details on the Laplace transform of the Basset-Boussinesq-Oseen equation}

By explicit computation, the following relationship can be obtained from Eq. (3):

$$
\begin{aligned}
& \hat{K}_{1}(s)=\frac{\frac{1}{\mathrm{St}}}{s+\frac{1}{\mathrm{St}}+\sqrt{\frac{3 \beta s}{\mathrm{St}}}}=\frac{\frac{1}{\mathrm{St}}}{\left(\sqrt{s}+A_{1}\right)\left(\sqrt{s}+A_{2}\right)} \\
= & \frac{1}{\operatorname{St}\left(A_{2}-A_{1}\right)}\left(\frac{1}{\sqrt{s}+A_{1}}-\frac{1}{\sqrt{s}+A_{2}}\right) .
\end{aligned}
$$

Note that the last step is valid if and only if $A_{1} \neq A_{2}$, where:

$$
\begin{aligned}
& A_{1}=\frac{1}{2}\left[\sqrt{\frac{3 \beta}{\mathrm{St}}}-\sqrt{\frac{3 \beta}{\mathrm{St}}-\frac{4}{\mathrm{St}}}\right] \\
& A_{2}=\frac{1}{2}\left[\sqrt{\frac{3 \beta}{\mathrm{St}}}+\sqrt{\frac{3 \beta}{\mathrm{St}}-\frac{4}{\mathrm{St}}}\right]
\end{aligned}
$$


It is worth noting the occurrence of a bifurcation when the arguments in each of the second square roots become zero, that is $A_{1}=A_{2}=1 / \sqrt{\mathrm{St}}$ and $3 \beta=4$. This occurs when $\beta$ reaches the critical value:

$$
\beta_{c}=\frac{4}{3}
$$

For any larger $\beta, A_{1}$ and $A_{2}$ are always real and positive. In this case, we can get the expression of $K_{1}(t)$ by exploiting that the Laplace transform of $1 / \sqrt{s}+a$ iff $a>0, \quad a \in \mathbb{R}$ is :

$$
\frac{1}{\sqrt{\pi t}}-a e^{a^{2} t} \operatorname{erfc}(a \sqrt{t})(s)
$$

This fact can be easily checked by direct calculation. The expression of $K_{1}(t)$ thus immediately follows:

$$
K_{1}(t)=\frac{1}{\operatorname{St}\left(A_{2}-A_{1}\right)}\left(A_{2} e^{A_{2}^{2} t} \operatorname{erfc}\left(A_{2} \sqrt{t}\right)-A_{1} e^{A_{1}^{2} t} \operatorname{erfc}\left(A_{1} \sqrt{t}\right)\right),
$$

indicating the complementary Euler error function by erfc:

$$
\operatorname{erfc}(a \sqrt{t})=1-\frac{2}{\sqrt{\pi}} \int_{0}^{a \sqrt{t}} e^{-r^{2}} d r=1-\frac{a}{\sqrt{\pi}} \int_{0}^{t} \frac{e^{-a^{2} r}}{\sqrt{r}} d r
$$

It is thus easy to see that Eq. (A4) is the correct antitransform when $A_{1}, A_{2}$ are real and positive. At the critical value of $\beta$, we can still try to obtain the expression of the Green function by taking the limit $A_{1} \rightarrow A_{2}$ in Eq. (A4). The coefficients before any term in Eq. (1) suggest that, from a physical point of view, at the critical $\beta$ there is a balance between Stokes drag and Basset history force. Above this value, the Basset term overcomes the drag friction. The opposite occurs for $\beta$ below the critical value, for which $A_{1}$ and $A_{2}$ become complex conjugates. Previous formula (A4) can be analytically extended to the complex case too. Notice that, from Eq. (A5), if and only if $\Re a^{2}>0$ we have $\lim _{t \rightarrow \infty} \operatorname{erfc}(a \sqrt{t})=0$, by virtue of the fact that

$$
\int_{0}^{+\infty} e^{-a^{2} x^{2}}=\frac{\sqrt{\pi}}{2 a}
$$

only when $\Re a^{2}>0$ or $\Re a^{2}=0$ but $\Im a^{2} \neq 0$ (here $\Re$ and $\Im$ denote the real and immaginary part of a complex number, respectively). If that happens, $\sqrt{a^{2}}$ is meant on the sub-branch such that the argument of $a$ is in the interval $[-\pi / 4, \pi / 4]$, the role of $a$ being played by either $A_{1}$ or $A_{2}$.

The above mentioned bifurcation also occurs for the Green function $\hat{K}_{0}(s)$, since its denominator is the same as the denominator of $\hat{K}_{1}(s)$. 


\section{Appendix B: Details on the eddy diffusivity for shear flows}

To begin, let us consider the exponent of Eq. (18). If one writes the integrals with respect to the endpoints which the Heaviside function specifies, that expression can be recast as:

$$
\int_{0}^{t_{1}} d s\left[G\left(t_{1}-s\right)\right]^{2}+\int_{0}^{t_{2}} d s\left[G\left(t_{2}-s\right)\right]^{2}-2 \int_{0}^{\min \left(t_{2}, t_{1}\right)} d s G\left(t_{1}-s\right) G\left(t_{2}-s\right)
$$

Let us now suppose that $t_{2}=t_{1}+t>t_{1}$. After changing variables, Eq. (B1) can be arranged as:

$$
2 \int_{0}^{t_{1}} d s[G(s)]^{2}+\int_{t_{1}}^{t+t_{1}} d s[G(s)]^{2}-2 \int_{0}^{t_{1}} d s G(s) G(s+t)
$$

and we get the stationary asymptotic limit through $t_{1} \rightarrow \infty$. The function $G(s)$ is easily computable by integrating Eq. (7) by parts:

$$
G(s)=\frac{A_{1}-A_{2}+A_{2} e^{A_{1}^{2} s} \operatorname{erfc}\left(A_{1} \sqrt{s}\right)-A_{1} e^{A_{2}^{2} s} \operatorname{erfc}\left(A_{2} \sqrt{s}\right)}{\operatorname{St} A_{1} A_{2}\left(A_{1}-A_{2}\right)}
$$

From Eq. (B3), and taking into account Eqs. (A5-A6), since $\operatorname{erfc}(a \sqrt{x})$ tends to 0 when $\Re a^{2} \geq 0$, it is easy to see that:

$$
\lim _{s \rightarrow \infty} G(s)=\frac{1}{A_{1} A_{2} \mathrm{St}}=1,
$$

whenever $\Re A_{1}^{2} \Re A_{2}^{2} \geq 0$. In the other case $\Re A_{1}^{2}, \Re A_{2}^{2}<0$, for the foregoing limit to be verified one can rewrite Eq. (B3) by exploiting again Eq. (A5):

$$
\begin{aligned}
G(t) & =\frac{1}{2 i A_{1} A_{2} \Im A_{2} \mathrm{St}}\left[2 i \Im A_{2}-A_{2} A_{1} e^{A_{1}^{2} t}+A_{1} A_{2} e^{A_{2}^{2} t}\right] \\
& +\frac{1}{2 i \Im A_{2} \mathrm{St}}\left[\frac{1}{\sqrt{\pi}} \int_{0}^{t} \frac{e^{-A_{1}^{2}(r-t)}-e^{-A_{2}^{2}(r-t)}}{\sqrt{r}} d r\right] \\
& =\frac{1}{A_{1} A_{2} \mathrm{St}}-\frac{1}{2 i \Im A_{2} \mathrm{St}}\left[e^{A_{1}^{2} t}-e^{A_{2}^{2} t}-\frac{1}{\sqrt{\pi}} \int_{0}^{t} \frac{e^{A_{1}^{2} r}-e^{A_{2}^{2} r}}{\sqrt{r+t}} d r\right]
\end{aligned}
$$

The last integral goes to zero at long time because:

$$
0 \leq \int_{0}^{t}\left|\frac{e^{A_{1}^{2} r}-e^{A_{2}^{2} r}}{\sqrt{r+t}}\right| d r \leq \frac{1}{\sqrt{t}} \int_{0}^{t}\left[\left|e^{A_{1}^{2} r}\right|+\left|e^{A_{2}^{2} r}\right|\right] d r=\frac{M}{\sqrt{t}} \underset{t \rightarrow \infty}{\longrightarrow} 0
$$


since the integral over the absolute value of the complex exponentials are convergent. The same exponentials decay to zero in the first square brackets of Eq. (B5) and, as a result of this, one recovers the same limit as the real case (B4) when $t$ tends to infinity.

We can therefore conclude that the second integral in Eq. (B2) tends to $t$, because the integrating function tends to 1 when its argument becomes large. We also see that $K_{1}(t) \rightarrow 0$ as $t \rightarrow \infty$ and, as to the initial condition term in Eq.(6), we have $\boldsymbol{\sigma}\left(\boldsymbol{\xi}_{0}, \boldsymbol{v}_{0}, t\right) \rightarrow 0$.

\section{Appendix C: Explicit real expressions for the kernel $K_{1}(t)$}

We are going to show in this Appendix the explicit real form of Eq. (7) below the bifurcation point. Real expressions might be easier to handle when one implements them in a code or integrates them numerically.

\section{Real case $\left(\beta>\beta_{c}\right)$}

When $A_{1}, A_{2} \in \mathbb{R}$, we can use Eq. (7). We will also need:

$$
\operatorname{erfc}(x)=1-\operatorname{erf}(x)
$$

where $\operatorname{erf}(x) \rightarrow 1$ when $x \rightarrow \infty$.

\section{Complex case $\left(\beta<\beta_{c}\right)$}

Let us move to discuss the case when $A_{1}, A_{2} \notin \mathbb{R}$. In this case, it is instructive to rewrite the analytical continuations of the Euler functions in Eq. (7) so as to emphasise their purely real and oscillating nature. To start, $A_{2}=\Re A_{2}+i \Im A_{2}$ and $A_{1}=\Re A_{2}-i \Im A_{2}$.Also, 


$$
\begin{aligned}
A_{2}^{2}= & \left(\Re A_{2}\right)^{2}-\left(\Im A_{2}\right)^{2}+2 i \Im A_{2} \Re A_{2} \text { and } A_{1}^{2}=\left(\Re A_{2}\right)^{2}-\left(\Im A_{2}\right)^{2}-2 i \Im A_{2} \Re A_{2} . \text { We get: } \\
K_{1}(t) & =\frac{1}{2 i \Im A_{2} \mathrm{St}}\left[A_{2} e^{A_{2}^{2} t}-A_{1} e^{A_{1}^{2} t}\right] \\
& -\frac{1}{2 i \Im A_{2} \mathrm{St}}\left[\frac{1}{\sqrt{\pi}} \int_{0}^{t} \frac{A_{2}^{2} e^{-A_{2}^{2}(r-t)}-A_{1}^{2} e^{-A_{1}^{2}(r-t)}}{\sqrt{r}} d r\right] \\
& =\frac{1}{\mathrm{St}}\left[\frac{\Re A_{2}}{\Im A_{2}} e^{\Re A_{2}^{2} t} \sin \left(\Im A_{2}^{2} t\right)+e^{\Re A_{2}^{2} t} \cos \left(\Im A_{2}^{2} t\right)\right] \\
& -\frac{1}{\Im A_{2} \mathrm{St}}\left[\frac{1}{\sqrt{\pi}} \int_{0}^{t} \frac{\Re A_{2}^{2} e^{\Re A_{2}^{2}(t-r)} \sin \left(\Im A_{2}^{2}(t-r)\right)+\Im A_{2}^{2} e^{\Re A_{2}^{2}(t-r)} \cos \left(\Im A_{2}^{2}(t-r)\right)}{\sqrt{r}} d r\right]
\end{aligned}
$$

Finally, in terms of $\beta$ and St:

$$
\begin{aligned}
K_{1}(t) & =\frac{e^{\left(\frac{3 \beta}{2}-1\right) \frac{t}{\mathrm{St}}}}{\mathrm{St}}\left[\frac{1}{\sqrt{\frac{4}{3 \beta}-1}} \sin \left(\sqrt{3 \beta} \sqrt{1-\frac{3 \beta}{4}} \frac{t}{\mathrm{St}}\right)+\cos \left(\sqrt{3 \beta} \sqrt{1-\frac{3 \beta}{4}} \frac{t}{\mathrm{St}}\right)\right] \\
& -\frac{1}{\sqrt{1-\frac{3 \beta}{4}} \mathrm{St}^{3 / 2}} \frac{1}{\sqrt{\pi}} \int_{0}^{t} d r \frac{e^{\left(\frac{3 \beta}{2}-1\right) \frac{r}{\mathrm{St}}}}{\sqrt{t-r}} \\
& \times\left[\left(\frac{3 \beta}{2}-1\right) \sin \left(\sqrt{3 \beta} \sqrt{1-\frac{3 \beta}{4}} \frac{r}{\mathrm{St}}\right)+\left(\sqrt{3 \beta} \sqrt{1-\frac{3 \beta}{4}}\right) \cos \left(\sqrt{3 \beta} \sqrt{1-\frac{3 \beta}{4}} \frac{r}{\mathrm{St}}\right)\right]
\end{aligned}
$$

It is worthy pointing out that, when we take the heavy-particle limit $\beta=0$, we recover the correct limit showed in ${ }^{40}$ :

$$
K_{1}(t)=\frac{e^{-\frac{t}{\mathrm{St}}}}{\mathrm{St}}
$$

When $\Re A_{1}^{2}, \Re A_{2}^{2}>0$, however, it is more convenient to exploit that:

$$
\operatorname{erfc}(a \sqrt{t}):=\frac{2}{\sqrt{\pi}} \int_{a \sqrt{t}}^{\infty} e^{-r^{2}} d r=\frac{a}{\sqrt{\pi}} \int_{t}^{\infty} \frac{e^{-a^{2} r}}{\sqrt{r}} d r, \Re a^{2}>0
$$

The conditions of the square is necessary to guarantee the convergence of the Gaussian integrals. This happens when, besides $\beta<\beta_{c}$ :

$$
3 \beta>2 \Rightarrow \beta>\bar{\beta}=\frac{2}{3}
$$


If the foregoing conditions are fulfilled, it follows that:

$$
\begin{aligned}
K_{1}(t) & =\frac{1}{\operatorname{St}\left(A_{2}-A_{1}\right)} \frac{1}{\sqrt{\pi}} \int_{t}^{\infty} d r \frac{A_{2}^{2} e^{-A_{2}^{2}(r-t)}-A_{1}^{2} e^{-A_{1}^{2}(r-t)}}{\sqrt{r}} \\
& =\frac{1}{\sqrt{1-\frac{3 \beta}{4}} \mathrm{St}^{3 / 2}} \frac{1}{\sqrt{\pi}} \int_{0}^{\infty} d r \frac{e^{-\left(\frac{3 \beta}{2}-1\right) \frac{r}{\mathrm{St}}}}{\sqrt{r+t}} \\
& \times\left[\left(\frac{3 \beta}{2}-1\right) \sin \left(\sqrt{3 \beta} \sqrt{1-\frac{3 \beta}{4}} \frac{r}{\mathrm{St}}\right)\right. \\
& \left.+\left(\sqrt{3 \beta} \sqrt{1-\frac{3 \beta}{4}}\right) \cos \left(\sqrt{3 \beta} \sqrt{1-\frac{3 \beta}{4}} \frac{r}{\mathrm{St}}\right)\right]
\end{aligned}
$$

At the threshold $\beta \rightarrow \bar{\beta}$ the exponential decay in the integral is suppressed and $K_{1}(t)$ takes the following very simple form:

$$
\left.K_{1}(t)\right|_{\beta=\bar{\beta}}=\frac{1}{\mathrm{St}^{3 / 2}} \sqrt{\frac{2}{\pi}} \int_{0}^{\infty} d r \frac{\cos \frac{r}{\mathrm{St}}}{\sqrt{r+t}}
$$

\section{Appendix D: Generation of a shear, incompressible, stochastic flow with oscillating autocorrelation in time}

We hereby present a stochastic method to generate a parallel flow whose Eulerian correlation function has a single wavenumber and decays in time like Eq. (22). Given N particles to average over, we want to generate a stochastic flow for each particle $p$ of the shape:

$$
\boldsymbol{u}_{p}=\left(A_{p}(t) \cos \left(\boldsymbol{k}_{0} \cdot \boldsymbol{x}+\phi_{p}\right), 0\right)
$$

Each $p$-th particle will see a different $A_{p}(t)$ and static phase $\phi_{p}$. Such a flow will be statistically homogeneous ${ }^{44}$. The energy of the flow in the asymptotic stationary limit

$$
E=\lim _{t \rightarrow \infty} \frac{1}{2}\left\langle u_{1}^{2}(t)\right\rangle
$$

and the sole non-null component $C_{11}(t)$ of its correlation tensor

$$
\mathbb{C}(t)=\lim _{t^{\prime} \rightarrow \infty}\left\langle u_{1}\left(t+t^{\prime}\right) u_{1}\left(t^{\prime}\right)\right\rangle
$$

are evaluated averaging the quantities in the brackets over the $\mathrm{N}$ particles. 
In the case of pure exponential correlation, the amplitude of the flow in Eq. (D1) corresponds to an Orstein-Uhlenbeck process with variance equal to 4 and unity decay time ${ }^{24}$ :

$$
d A_{i}(t)=-A_{i}(t) d t+\sqrt{8 E} d \omega(t)
$$

where $E=\left\langle A^{2}\right\rangle / 4$ is the (asymptotically) stationary variance. Seeing that we want to have a further oscillating contribution with angular frequency $\Omega$, the above process needs to be complexified and leveraged to get the following SDE:

$$
d A(t)=-A(t)(1-\imath \Omega) d t+\sqrt{2\left\langle(\Re A)^{2}\right\rangle}\left[d \omega_{1}(t)+\imath d \omega_{2}(t)\right]
$$

where $d \omega_{1}(t), d \omega_{2}(t)$ are two independent Wiener processes and $\left\langle(\Re A)^{2}\right\rangle=4 E$. All we need is the real part of the solution of this complex SDE:

$$
\begin{aligned}
\Re A(t) & =\Re A\left(t_{0}\right) e^{-t} \cos (\Omega t)-\Im A\left(t_{0}\right) e^{-t} \sin (\Omega t) \\
& +\sqrt{8 E}\left[\int_{t_{0}}^{t} e^{-\left(t-t_{0}\right)} \cos \left(\Omega\left(t-t_{0}\right)\right) d \omega_{1}(t)-\int_{t_{0}}^{t} e^{-\left(t-t_{0}\right)} \sin \left(\Omega\left(t-t_{0}\right)\right) d \omega_{2}(t)\right]
\end{aligned}
$$

The mean value of the process above is manifestly zero. By recalling the well- known trigonometric Werner formulae, if $A\left(t_{0}\right)=0$ or, equivalently, if one waits a suitable thermalization time $\gg 1$ for an arbitrary initial condition, we get the following expression:

$$
\left\langle\Re A\left(t_{1}\right) \Re A\left(t_{2}\right)\right\rangle=4 E e^{-\left|t_{1}-t_{2}\right|} \cos \left(\Omega\left(t_{1}-t_{2}\right)\right)
$$

\section{REFERENCES}

${ }^{1}$ L. E. Reichl, A Modern Course in Statistical Physics, 4th ed. (Wiley,, 2016).

${ }^{2}$ T. F. Stocker, D. Qin, G.-K. Plattner, M. Tignor, S. Allen, J. Boschung, A. Nauels, Y. Xia, V. Bex, and P. M. Midgley (eds.), "Ipcc, 2013: Climate change 2013: The physical science basis. contribution of working group i to the fifth assessment report of the intergovernmental panel on climate change." (2013).

${ }^{3}$ A. Bracco, P. H. Chavanis, A. Provenzale, and E. A. Spiegel, "Particle aggregation in a turbulent keplerian flow," Physics of Fluids 11, 2280-2287 (1999), https://doi.org/10.1063/1.870090.

${ }^{4}$ M. Wilkinson, B. Mehlig, and V. Uski, "Stokes trapping and planet formation," The Astrophysical Journal Supplement Series 176, 484-496 (2008). 
${ }^{5}$ G. Falkovich, A. Fouxon, and M. G. Stepanov, "Acceleration of rain initiation by cloud turbulence," Nature 419, 151-154 (2002).

${ }^{6}$ A. Celani, G. Falkovich, A. Mazzino, and A. Seminara, "Droplet condensation in turbulent flows," Europhysics Letters (EPL) 70, 775-781 (2005).

${ }^{7}$ A. Celani, A. Mazzino, and M. Tizzi, "The equivalent size of cloud condensation nuclei," New Journal of Physics 10, 075021 (2008).

${ }^{8}$ D. S. Lemons and D. L. Kaufman, "Brownian motion of a charged particle in a magnetic field," IEEE Transactions on Plasma Science 27, 1288-1296 (1999).

${ }^{9}$ R. Czopnik and P. Garbaczewski, "Brownian motion in a magnetic field," Phys. Rev. E 63, 021105 (2001).

${ }^{10}$ G. Eslami, E. Esmaeilzadeh, and A. T. Pérez, "Modeling of conductive particle motion in viscous medium affected by an electric field considering particle-electrode interactions and microdischarge phenomenon," Physics of Fluids 28, 107102 (2016), https://doi.org/10.1063/1.4964683.

${ }^{11}$ I. Fouxon and A. Leshansky, "Phytoplankton's motion in turbulent ocean," Phys. Rev. E 92, 013017 (2015).

${ }^{12} \mathrm{C} . \mathrm{M}$. Tchen, Mean value and correlation problems connected with the motion of small particles suspended in a turbulent fluid, Ph.D. thesis, University of Delft (1947).

${ }^{13}$ E. E. Michaelides, "Hydrodynamic force and heat/mass transfer from particles, bubbles, and drops," Journal of Fluids Engineering 125, 209-238 (2003).

${ }^{14}$ M. R. Maxey and J. J. Riley, "Equation of motion for a small rigid sphere in a nonuniform flow," The Physics of Fluids 26, 883-889 (1983), https://aip.scitation.org/doi/pdf/10.1063/1.864230.

${ }^{15}$ L. Bergougnoux, G. Bouchet, D. Lopez, and Guazzelli, "The motion of solid spherical particles falling in a cellular flow field at low stokes number," Physics of Fluids 26, 093302 (2014), https://doi.org/10.1063/1.4895736.

${ }^{16}$ T. Sapsis and G. Haller, "Inertial particle dynamics in a hurricane," Journal of the Atmospheric Sciences 66, 2481-2492 (2009), https://doi.org/10.1175/2009JAS2865.1.

${ }^{17} \mathrm{~F}$. Toschi and E. Bodenschatz, "Lagrangian properties of particles in turbulence," Annual Review of Fluid Mechanics 41, 375-404 (2009), https://doi.org/10.1146/annurev.fluid.010908.165210.

${ }^{18}$ P. Monroy, E. Hernández-García, V. Rossi, and C. López, "Modeling the dynamical 
sinking of biogenic particles in oceanic flow," Nonlinear Processes in Geophysics 24, 293305 (2017).

${ }^{19}$ Q. Dai, T. Jin, K. Luo, and J. Fan, "Direct numerical simulation of particle dispersion in a three-dimensional spatially developing compressible mixing layer," Physics of Fluids 30, 113301 (2018), https://doi.org/10.1063/1.5054744.

${ }^{20}$ D. H. Richter, "Turbulence modification by inertial particles and its influence on the spectral energy budget in planar couette flow," Physics of Fluids 27, 063304 (2015), https://doi.org/10.1063/1.4923043.

${ }^{21}$ S. Murray, M. F. Lightstone, and S. Tullis, "Single-particle lagrangian and structure statistics in kinematically simulated particle-laden turbulent flows," Physics of Fluids 28, 033302 (2016), https://doi.org/10.1063/1.4942815.

${ }^{22}$ S. Boi, A. Mazzino, P. Muratore-Ginanneschi, and S. Olivieri, "Generalization of taylor's formula to particles of arbitrary inertia," Phys. Rev. Fluids 3, 104501 (2018).

${ }^{23}$ A. S. Berrouk, Stochastic Lagrangian Modeling for Large Eddy Simulation of Dispersed Turbulent Two-Phase Flows (Bentham Science Publishers (February 15, 2018), 2018).

${ }^{24}$ S. Boi, A. Mazzino, and G. Lacorata, "Explicit expressions for eddy-diffusivity fields and effective large-scale advection in turbulent transport," Journal of Fluid Mechanics 795, 524-548 (2016).

${ }^{25}$ G. Lacorata, A. Mazzino, and U. Rizza, "3d chaotic model for subgrid turbulent dispersion in large eddy simulations," Journal of the Atmospheric Sciences 65, 2389-2401 (2008).

${ }^{26}$ J. C. H. Fung, J. C. R. Hunt, N. A. Malik, and R. J. Perkins, "Kinematic simulation of homogeneous turbulence by unsteady random fourier modes," Journal of Fluid Mechanics 236, 281-318 (1992).

${ }^{27}$ T. P. Sapsis, N. T. Ouellette, J. P. Gollub, and G. Haller, "Neutrally buoyant particle dynamics in fluid flows: Comparison of experiments with lagrangian stochastic models," Physics of Fluids 23, 093304 (2011), https://doi.org/10.1063/1.3632100.

${ }^{28}$ S. Tenneti, M. Mehrabadi, and S. Subramaniam, "Stochastic lagrangian model for hydrodynamic acceleration of inertial particles in gas-solid suspensions," Journal of Fluid Mechanics 788, 695-729 (2016).

${ }^{29}$ W. Tang, B. Knutson, A. Mahalov, and R. Dimitrova, "The geometry of inertial particle mixing in urban flows, from deterministic and random displacement models," Physics of Fluids 24, 063302 (2012), https://doi.org/10.1063/1.4729453. 
${ }^{30}$ A. Mazzino and M. Vergassola, "Interference between turbulent and molecular diffusion," Europhysics Letters (EPL) 37, 535-540 (1997).

${ }^{31}$ A. Sarracino, F. Cecconi, A. Puglisi, and A. Vulpiani, "Nonlinear response of inertial tracers in steady laminar flows: Differential and absolute negative mobility," Phys. Rev. Lett. 117, 174501 (2016).

${ }^{32}$ P. Castiglione, A. Crisanti, A. Mazzino, M. Vergassola, and A. Vulpiani, "Resonant enhanced diffusion in time-dependent flow," Journal of Physics A: Mathematical and General 31, 7197-7210 (1998).

${ }^{33}$ O. A. Druzhinin and L. A. Ostrovsky, "The influence of basset force on particle dynamics in two-dimensional flows," Phys. D 76, 34-43 (1994).

${ }^{34}$ A. Daitche and T. Tél, "Memory effects are relevant for chaotic advection of inertial particles," Phys. Rev. Lett. 107, 244501 (2011).

${ }^{35}$ M. van Hinsberg, J. ten Thije Boonkkamp, and H. Clercx, "An efficient, second order method for the approximation of the basset history force," Journal of Computational Physics 230, 1465 - 1478 (2011).

${ }^{36}$ R. L. Honeycutt, "Stochastic runge-kutta algorithms. i. white noise," Phys. Rev. A 45, 600-603 (1992).

${ }^{37}$ U. Frisch, "Lecture on turbulence and lattice gas hydrodynamics," in Lecture Notes, NCAR-GTP Summer School June 198\%, edited by G. T. Rado and H. Suhl (J.R. Herring and J. C.McWilliams (World Scientific), 1987) pp. 219-371.

${ }^{38} \mathrm{M}$. W. Reeks, "The relationship between brownian motion and the random motion of small particles in a turbulent flow," The Physics of Fluids 31, 1314-1316 (1988), https://aip.scitation.org/doi/pdf/10.1063/1.866722.

${ }^{39}$ L. Biferale, A. Crisanti, M. Vergassola, and A. Vulpiani, "Eddy diffusivities in scalar transport," Physics of Fluids 7, 2725-2734 (1995), https://doi.org/10.1063/1.868651.

${ }^{40} \mathrm{~S}$. Boi, A. Mazzino, and P. Muratore-Ginanneschi, "Eddy diffusivities of inertial particles in random gaussian flows," Phys. Rev. Fluids 2, 014602 (2017).

${ }^{41}$ M. R. Maxey, "The motion of small spherical particles in a cellular flow field," The Physics of Fluids 30, 1915-1928 (1987), https://aip.scitation.org/doi/pdf/10.1063/1.866206.

${ }^{42}$ U. Frisch, Turbulence: The Legacy of A. N. Kolmogorov (Cambridge University Press, 1995).

${ }^{43}$ G. I. Taylor, "Diffusion by Continuous Movements," Proceedings of the London Math- 
ematical Society s2-20, 196-212 (1922), http://oup.prod.sis.lan/plms/article-pdf/s220/1/196/4349546/s2-20-1-196.pdf.

${ }^{44} \mathrm{~S}$. Boi, M. M. Afonso, and A. Mazzino, "Anomalous diffusion of inertial particles in random parallel flows: theory and numerics face to face," Journal of Statistical Mechanics: Theory and Experiment 2015, P10023 (2015).

${ }^{45}$ Mazzino, A. and Castiglione, P., "Interference phenomena in scalar transport induced by a noise finite correlation time," Europhys. Lett. 45, 476-481 (1999). 